\title{
Dynamical properties of Ultraluminous Infrared Galaxies. II. Traces of dynamical evolution and end products of local ultraluminous mergers ${ }^{1}$
}

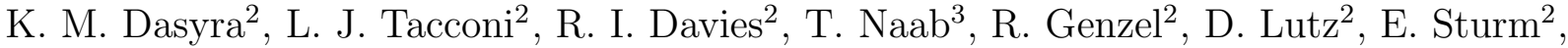 \\ A. J. Baker ${ }^{4,5}$, S. Veilleux ${ }^{5}$, D. B. Sanders ${ }^{6}$, A. Burkert ${ }^{3}$
}

\begin{abstract}
We present results from our Very Large Telescope large program to study the dynamical evolution of local Ultraluminous Infrared Galaxies (ULIRGs) and QSOs. This paper is the second in a series presenting the stellar kinematics of 54 ULIRGs, derived from high resolution, long-slit $H$ - and $K$-band spectroscopy. The data presented here, including observations of 17 new targets, are mainly focused on sources that have coalesced into a single nucleus. The stellar kinematics, extracted from the CO ro-vibrational bandheads in our spectra, indicate that ULIRG remnants are dynamically heated systems with a mean dispersion of $161 \mathrm{~km} \mathrm{~s}^{-1}$. The combination of kinematic, structural, and photometric properties of the remnants indicate that they mostly originate from major mergers and that they result in the formation of systems supported by random motions, therefore, elliptical galaxies. The peak of the velocity dispersion distribution and the locus of ULIRGs on the fundamental plane of early-type galaxies indicate that the end products of ultraluminous mergers are typically moderate-mass ellipticals (of stellar mass $\sim 10^{10}$ $\left.10^{11} M_{\odot}\right)$. Converting the host dispersion into black hole mass with the aid of the $M_{\mathrm{BH}}-\sigma$ relation yields black hole mass estimates of the order $10^{7}-10^{8} M_{\odot}$ and high accretion rates with Eddington efficiencies often $>0.5$.
\end{abstract}

Subject headings: galaxies: formation — galaxies: kinematics and dynamics — infrared: galaxies — ISM: kinematics and dynamics -

\section{Introduction}

Galaxy mergers, which have a frequency increasing with redshift (e.g., Toomre 1977; Kauffmann \& White 1993; Le Fèvre et al. 2000), are considered a key mechanism in driving galaxy evolution. In the local Universe, the ultraluminous

\footnotetext{
${ }^{2}$ Max-Planck-Institut für extraterrestrische Physik, Postfach 1312, 85741, Garching, Germany

${ }^{3}$ Universitätssternwarte, Scheinerstr. $\quad 1, \quad 81679$, München, Germany

${ }^{4}$ Jansky Fellow, National Radio Astronomy Observatory

${ }^{5}$ Department of Astronomy, University of Maryland, College Park, MD 20742, USA

${ }^{6}$ Institute for Astronomy, University of Hawaii, 2680 Woodlawn Drive, Honolulu, HI 96822, USA

${ }^{1}$ Based on observations at the European Southern Observatory (ESO 171.B-044)
}

infrared galaxies (ULIRGs) are excellent laboratories for studying violent merging events. ULIRGs are mergers of gas-rich galaxies observed during strong starburst events; these episodes have durations $\lesssim 10^{8}$ yrs (e.g. Canalizo \& Stockton 2001; Mihos \& Hernquist 1996) and are brief compared to the baryonic matter merging process $\left(\sim 10^{9} \mathrm{yrs}\right.$; e.g. Barnes 1992; Hernquist 1993). The starburst emission, often combined with emission originating from an active galactic nucleus (AGN), gives rise to infrared (IR) luminosities greater than $10^{12} L_{\odot}$ (e.g., Sanders \& Mirabel 1996; Lonsdale et al. 2006 and references therein); these luminosities are comparable to the bolometric luminosities of QSOs.

A plethora of studies indicates that ULIRGs transform (gas-rich) spiral (S) galaxies into ellipti- 
cals (Es) through merger-induced dissipative collapse (Kormendy \& Sanders 1992; Mihos \& Hernquist 1996; Barnes \& Hernquist 1996). The large molecular gas concentrations in the central kpc regions of ULIRGs (e.g. Downes \& Solomon 1998; Bryant \& Scoville 1999) have densities comparable to stellar densities in ellipticals. The light profiles of ULIRG remnants often follow a $\mathrm{r}^{1 / 4}$ law (e.g., Wright et al. 1990; Stanford \& Bushouse 1991; James et al. 1999; Scoville et al. 2000; Rothberg \& Joseph 2004). Kim et al. (2002) and Veilleux et al. (2002) established the solidity of this result by proving that the majority $(73 \%)$ of all single-nucleus ULIRGs in a sample of 118 sources with $60 \mu \mathrm{m}$ flux greater than 1 Jy (hereafter the 1 Jy sample; Kim \& Sanders 1998) have elliptical-like light profiles. Genzel et al. (2001) and Tacconi et al. (2002) have made high-resolution near-infrared (NIR) spectroscopic measurements of the stellar dynamics of small samples consisting mostly of fully-merged ULIRGs. They conclude that ULIRGs resemble intermediate mass ellipticals/lenticulars with moderate rotation, in terms of their velocity dispersion distribution, their location in the fundamental plane (FP; e.g., Djorgovski \& Davis 1987; Dressler et al. 1987) and their distribution of the rotation/velocity dispersion ratio. Together, these results suggest that ULIRGs form moderate mass ellipticals (of stellar mass $\sim 10^{11} \mathrm{M}_{\odot}$ ).

One way to investigate the physical details and the evolution of ULIRGs is to determine the kinematic and structural properties of the merging galaxies in different merger phases. We therefore conducted a European Southern Observatory (ESO) large program ${ }^{2}$ that traces the host dynamics of a large sample of ULIRGs spanning wide ranges of merger phase and infrared luminosity through NIR spectroscopy. Our study extends the previous work of Genzel et al. (2001) and Tacconi et al. (2002). The enlarged sample comprises ULIRGs at wider ranges of merger phase and luminosity, and higher redshifts than those of Genzel et al. (2001) and Tacconi et al. (2002). The new observations of 38 sources increase the original sample by more than a factor of 3 , enhancing our statistics and enabling us to study the properties of galaxy mergers as a function of time. In

${ }^{2}$ (PI Tacconi)
Dasyra et al. (2006, hereafter Paper I) we analyzed those ULIRGs that are in a merger phase later than the first encounter but prior to nuclear coalescence, and hence, show more than one nucleus in the NIR acquisition images. In this paper we present results mainly from those ULIRGs that have coalesced and show a single nucleus in our images, the so-called remnants. We compare the stellar kinematic properties of binary ULIRGs and ULIRG remnants to look for traces of dynamical evolution in ultraluminous mergers.

Some of the ULIRGs presented in this study may in fact be binary sources very close to coalescence that have small projected nuclear separations; such sources cannot always be resolved or kinematically disentangled due to instrumental angular resolution constraints. At redshifts typical for the sources in our sample, the angular resolution achieved implies that any unresolved systems will have nuclear separations smaller than 1.5 kpc. Merger simulations (e.g. Mihos 1999; Mihos 2000) have shown that by the time the individual nuclei are separated by $\lesssim 1 \mathrm{kpc}$, the low moments of the stellar kinematics (rotational velocity and velocity dispersion) have almost reached their relaxation values. Therefore, the dynamical properties of all the sources we classify as remnants are representative of those at dynamical equilibrium.

This paper is arranged as follows. We briefly summarize the observations and data reduction methods and present the pre- and post- coalescence ULIRG samples in $\S 2$. After studying whether the kinematic properties of the merging galaxies evolve during the ultraluminous merger phases in $\S 3$, we investigate the origins and the potential end products of ultraluminous mergers in $\S 4$ and $\S 5$ respectively. We then focus on the black holes hosted by ULIRGs: an analysis of the evolution of the $M_{\mathrm{BH}^{-}} \sigma$ relation during the merger is followed by a discussion of the nuclear activity implied by our data in $\S 6$. Finally a summary is presented in $\S 7$.

\section{Observations And Data Reduction}

\subsection{Data Acquisition And Analysis}

We combine the sources in our Very Large Telescope (VLT) program with the sources presented in Genzel et al. (2001) and Tacconi et al. (2002) to compile a sample that comprises 54 ULIRGs 
and 12 QSOs. The stellar kinematics of the QSOs will be presented in a forthcoming paper (Dasyra et al. 2006, in preparation). In this paper, we perform a study of the stellar kinematics in postcoalescence ultraluminous mergers (ULIRG remnants), after presenting new data for 17 ULIRGs and summarizing pre-existing data for 13 mainly post-coalescence sources. Of the 54 ULIRGs observed in total, 30 are confirmed to have two nuclei $^{3}$, and 1 (IRAS 00199-7426: Duc et al. 1997; Paper I) may be a multiple-interaction system. A detailed description of the criteria set to select these 54 sources from the $1 \mathrm{Jy}$ catalog is given in Paper I.

Our new high-resolution, long-slit spectroscopic data were obtained using the ISAAC spectrometer (Moorwood et al. 1998) mounted on the Antu telescope unit of the VLT. The observations were made in the $H$ and $K$ bands, with an instrument resolution of $\lambda / \delta \lambda=5100$ and $\lambda / \delta \lambda=4400$ respectively. The slit length was $120^{\prime \prime}$ and the slit width 0 ". 6 ; the detector scale was 0 .' 146 per pixel. The total exposure times and the slit position angles for each source are presented in Table 1. If the position angle of the major axis of rotation could not be identified for the remnants (e.g. from the elongation of the stellar disk), the slits were typically placed at $0^{\circ}$ and $90^{\circ}$. The redshift range of all sources in our sample is $0.018<z<0.268$ (see Table 1). No sources are observed for the redshift sub-range $0.163<z<0.199$, since the CO bandheads are then shifted into wavebands of high atmospheric absorption.

As in Paper I, we derive the structural parameters of the sources that do not have high-resolution NIR imaging (e.g. Hubble Space Telescope NICMOS) data by fitting ellipses to our 10-secondlong acquisition images. The acquisition images have also been obtained with the ISAAC detector, in its $H$-band imaging mode, which corresponds to a scale of $0^{\prime \prime} 147$ per pixel. We fit the ellipses with the aid of the SExtractor package (Bertin \& Arnouts 1996) and we present the half-light radius $R_{\text {eff }}{ }^{4}$, the ellipticity, and the angle $\phi_{\alpha}$ between the major axis of rotation and the position angle of the first slit in Table 2 for each source. To con-

\footnotetext{
${ }^{3} 20$ were first presented in Paper I and 10 are presented or summarized here; see the Appendix

${ }^{4}$ As measured from the ellipse that encloses half the total counts.
}

vert all angular distances into linear sizes we use a $H_{0}=70 \mathrm{~km} \mathrm{~s}^{-1} \mathrm{Mpc}^{-1}, \Omega_{m}=0.3, \Omega_{\text {total }}=1$ cosmology. For some of the sources in our sample, a measurement of the $K$-band effective radius is available in the literature (see Table 2). In these cases, we use the average NIR $R_{\text {eff value in all com- }}$ putations and diagrams that follow. The imaging analyses of Surace \& Sanders (1999) and Scoville et al. (2000) have shown that the difference between the measurement of $R_{\text {eff }}$ in ULIRGs in the $H$ and in the $K$ band is small and non-systematic. Therefore, averaging these NIR $R_{\text {eff }}$ values yields a measurement of higher accuracy.

The extraction of the stellar central velocity dispersion $\sigma$ and rotational velocity $V_{\text {rot }}$ from the spectra follows the method presented in Paper I. It is performed using the Fourier quotient technique (Bender 1990); this method provides the intrinsic line-of-sight (LOS) velocity profile along any given aperture. To this we fit a high-order Gaussian (linear combination of a Gaussian and a second order polynomial) to determine the average LOS radial velocity and velocity dispersion. The fit is performed to each bandhead individually and the errors are equal to the standard deviation of all measurements performed. The central aperture spectrum of each source, combined over the two slits and shifted to restframe, is displayed in Fig. 1. The stellar template that fits best the post-coalescence ULIRG spectra is an MOIII giant (HD 25472; presented in Genzel et al. 2001). In general, template mismatch affects the accuracy to which the stellar velocities are measured; we found that the difference in the velocity dispersion between the (best-fitting) M0III giant and the M1I supergiant (HD 99817; Genzel et al. 2001) is $\sim 15 \mathrm{~km} \mathrm{~s}^{-1}$. The M0III template is overplotted with a dashed line in Fig. 1, after being convolved with the Gaussian that best simulates the LOS broadening function.

In all of this paper's analyses we deem aperture effects to be negligible for the measurement of the stellar velocity dispersion. According to the merger simulations of Bendo \& Barnes (2000), the velocity dispersion in (relaxed) remnants may vary at most by $10 \%$ for the apertures considered here (up to $\sim 2 R_{\text {eff }}$ ) which are selected to maximize the signal-to-noise ratio. Resolution effects further reduce differences in the velocity dispersion measurement between various apertures. 
The LOS rotational velocity, $V_{\text {rot }}(\mathrm{LOS})$, is measured from apertures that exclude the center of the galaxy. The center and the annular width of the apertures used are tabulated in Table 3. For each slit, the observed rotational velocity $V_{\text {rot }}(o b s)$ can be derived from the LOS rotational velocity by correcting for the angular deviation of the slit from the major axis of rotation as

$$
V_{\text {rot }}(o b s)=V_{\text {rot }}[\mathrm{LOS}] / \cos \left[\phi_{\alpha}\right] \text {. }
$$

When rotation is observed along both slits, i.e. when none of the slits is very close to being perpendicular to the major axis of rotation, we average the results of the two slits. The value of the observed rotational velocity is given in Table 3 and is related to the actual rotational velocity $V_{\text {rot }}$ by

$$
V_{\text {rot }}(\mathrm{obs})=V_{\text {rot }} \sin (i)
$$

In this paper we do not compute the inclination $i$ from the ellipticity of each source (as we did in Paper I); this conversion is very uncertain for the ULIRG remnants since their stellar disks are dynamically hot (due to the advanced phase of the merger). Instead, we statistically determine the mean inclination that needs to be applied to the sample. For this purpose, we use the weighted mean value of $\sin (i)$ observed for disk-like galaxies on the sky. Since the probability $p[i]$ of finding a galaxy at an inclination $\mathrm{i}$ (in the range $\left[0^{\circ}, 90^{\circ}\right]$ ) scales with $\sin (i)$ (Collin et al. 2006), the mean value of $p[i] \sin (i)$ is $\pi / 4$. The stellar kinematic results (central velocity dispersion and rotational velocity) can be found in Table 3 .

\subsection{Pre- and Post-coalescence Sample Classification}

Prior to performing statistics on the kinematic parameters of the pre- and post- coalescence ULIRG samples, we descibe the criterion we use to compile the two samples; it consists of a nuclear separation threshold beyond which binary sources can be considered relaxed. According to simulations, this nuclear separation should be roughly 1 kpc (Mihos 2000). The actual value of the threshold we set depends on the resolution of the images we use to search for the presence of secondary components.

We compute the resolution of our images, which depends on the detector scale and the seeing, by averaging the FWHM of bright stars in each field. Our mean resolution corresponds to a FWHM of 4.3 pixels and enables us to resolve individual sources separated by $1 \mathrm{kpc}$ up to $z=0.080$. If we set the nuclear separation threshold to $1.5 \mathrm{kpc}$, we can distinguish individual sources up to $z=0.118$. The corresponding numbers of sources that are verified to satisfy the coalescence criterion are 10 and 23 , respectively. We adopt a nuclear separation cutoff of $1.5 \mathrm{kpc}$. For this nuclear separation, the low velocity moments are still close to their relaxation values according to the models of Mihos (2000) and Naab et al. (2006b).

Of the 7 sources at $z>0.118,5$ have been observed with the NICMOS camera onboard the Hubble Space Telescope by Veilleux et al. (2006) and Scoville et al. (2000). According to these authors, none of IRAS 003971312, IRAS 01572+0009, IRAS 04313-1649, IRAS $09039+0503$, and IRAS $14070+0525$ has a secondary object at distances $<1.5 \mathrm{kpc}$ that can be unambiguously characterized as a nucleus. For one of the remaining 2 sources, IRAS 23578-5307, the large elongation and the tidal tails indicate the possible presence of two components (see Fig. 2; left panel). To investigate this possibility, we deconvolve our acquisition image with its pointspread-function (PSF) which has a FWHM of 4.1 pixels. For this task, we use the LUCY algorithm of IRAF (Lucy 1974; Richardson 1972). The deconvolution increases the resolution of the image by a factor of $\sim 2$. The resulting image (see Fig. 2; right panel) confirms the presence of a second nucleus and constrains its projected separation to be $1.4 \pm 0.4 \mathrm{kpc}$. Unlike that of IRAS 23578 5307, the redshift of IRAS 11223-1244 is too high to enable us to derive any conclusions using image deconvolution techniques. IRAS 11223-1244 is therefore excluded from all statistics that follow. The same applies to the possibly multiple merger IRAS 00199-7426.

A summary of the classification of all sources in our sample can be found in Table 1 . We have a total sample of 29 ULIRG remnants, which consists of 21 single-nucleus or unresolved sources, and 8 confirmed close binaries that have nuclear separations less than or equal to the selected threshold. These sources namely are IRAS 00091-0738, IRAS 09111-1007, IRAS 14378-3651, IRAS 15250+3609, IRAS 23578-5307, 
Mrk 273, Arp 220 and NGC 6240 (see the Appendix). The pre-coalescence binary sample comprises 23 pairs of sources; of those, 40 individual components have velocity dispersion measurements.

\section{Traces of Evolution In The Stellar Kine- matics}

Taking into account the classification scheme of $\S 2.2$, we find that the $\sigma$ distribution of the 29 ULIRG remnants has a mean of $161 \mathrm{~km} \mathrm{~s}^{-1}$ and a median of $150 \mathrm{~km} \mathrm{~s}^{-1}$. The uncertainty in this mean, which is known as its standard error, is $8 \mathrm{~km} \mathrm{~s}^{-1}$ since the standard deviation equals $42 \mathrm{~km} \mathrm{~s}^{-1}$. For the 40 pre-coalescence ULIRG nuclei, we find that the mean is $142 \mathrm{~km} \mathrm{~s}^{-1}$, with a standard error of 3 and a standard deviation of 21 $\mathrm{km} \mathrm{s}^{-1}$, and the median is $145 \mathrm{~km} \mathrm{~s}^{-1}$. The preand post- coalescence dispersion distributions are shown in Fig. 3 (left panel). While the difference in the mean of the two distributions is small and the modes are the same, the remnant velocity dispersion distribution has a larger variance and a tail at the high- $\sigma$ end, the statistical significance of which needs to be quantified.

To investigate whether the two distributions are independent, we begin by using the KolmogorovSmirnov (KS) test, which makes no assumptions about the shape of the distributions under examination. We find that the maximum deviation $D$ between the cumulative distribution function of the two ULIRG populations is 0.285 , which for the number of sources we observed, corresponds to a probability of $89.4 \%$ that the populations are independent. This probability is below the widely accepted significance levels. However, a well-known disadvantage of the KS test is that it is sensitive to small number statistics. To address whether a better handling of small number statistics may alter our conclusions, we created Monte Carlo simulations.

The Monte Carlo code begins with the assumption that the two distributions are drawn from the same parent population. We simulated the parent distribution by spline interpolating between the observed $\sigma$ distribution of the combined preand post- coalescence ULIRGs (see Fig. 3, right panel). From the parent distribution, which consisted of $10^{6}$ points, we selected random points to generate two artificial progenitor/remnant subsamples. Each generated subsample had a number of elements equal to that of the corresponding real population. We repeated this procedure for 10000 iterations. In each iteration, we computed the difference in the mean of the two generated samples and the difference in the variance between each real population and the respective generated sample. At the end of all iterations, we counted how many times the difference in the mean of the two generated samples was equal to or greater than that measured, $19 \mathrm{~km} \mathrm{~s}^{-1}$. We found that it corresponded to a probability of $1.2 \%$. Similarly, the probability $p$ that the variances of both generated samples $\left(\sigma_{\mathrm{gen}, 1}^{2}\right.$ and $\sigma_{\mathrm{gen}, 2}^{2}$ respectively) are greater than those measured (or $p\left[\sigma_{\text {gen }, 1}>21 \mathrm{~km} \mathrm{~s}^{-1}\right] * p\left[\sigma_{\text {gen }, 2}>42 \mathrm{~km} \mathrm{~s}^{-1}\right]$ ) is $5.0 \%$. In other words, the different means imply a $98.8 \%$ probability that the two distributions are independent, while the different variances yield a $95.0 \%$ probability of independence. Since these probabilities correspond to $\sim 2.5$ and $\sim 2.0$ sigma respectively, the difference in the distributions is at or above the widely used significance levels. This result favors the hypothesis that the apparent kinematic evolution is real rather than an artifact of limited-number statistics.

Despite the large range of confidence levels indicated by the statistical tests, their conclusion can be summarized as follows: it is possible, although uncertain, that the observed increase in sigma reflects the dynamical heating of the merging galaxies. The importance of this result lies in that observations are able to constrain the dynamical heating that models predict for the ultraluminous phases of gas-rich mergers. Theory (Mihos \& Hernquist 1996; Mihos 1999; Springel et al. 2005) and observations (Mihos \& Bothun 1998; Murphy et al. 2001; Veilleux et al. 2002) show that strong starbursts (and associated ULIRG phases) in gas-rich mergers typically occur between first encounter and nuclear coalescence, altough in individual cases, their actual occurence may significantly vary in terms of time. On a statistical basis, a binary ULIRG sample is expected to be roughly mid-way from first encounter to coalescence; a ULIRG remnant sample is probably close to coalescence. For these merger phases, the theoretically predicted increase of the stellar velocity dispersion is small (Naab et al. 2006b); 
therefore observationally tracing it is very important. However, prior to comparing observations with models, further data are needed to quantify at 3 Gaussian-sigma levels the difference in $\sigma$ before and after nuclear coalescence.

Another possibility that needs to be investigated prior to comparing the evolution in the stellar kinematics between observations and models is whether the double- and single- nucleus ULIRGs may originate from mergers of galaxies of somewhat different mass contents: the ULIRGs that are prior to nuclear coalescence may be more gasrich (and possibly more massive) than the remnants, allowing them to have an ultraluminous phase of comparable IR output at earlier merger phases (Mihos \& Hernquist 1996). In this case, the observed dynamical heating would be less than what would be measured if we were able to observe a specific galaxy pair from the beginning until the end of the merging process. To investigate whether the starburst activity is triggered under similar conditions for pre- and post- coalescence ULIRGs, we need to quantify and compare the gas-mass content of both samples. Molecular gas mass measurements obtained by Gao \& Solomon (1999) in local Luminous Infrared Galaxies (LIRGs; sources of $10^{11} L_{\odot}<L_{\mathrm{IR}}<10^{12} L_{\odot}$ ) and ULIRGs indicate that a correlation between nuclear separation and gas fraction is observed in LIRGs but not in ULIRGs. To properly address the question, molecular-gas-content observations need to be performed on ULIRG samples with sizes comparable to those of the dynamical studies.

The mean value of the rotational velocity is 62 (and the standard deviation is 59 ) $\mathrm{km} \mathrm{s}^{-1}$ for the ULIRG remnants, increasing to $79 \mathrm{~km} \mathrm{~s}^{-1}$ when inclination effects are statistically accounted for. The observed stellar rotational velocity of each individual source and its ratio over the velocity dispersion is presented in Table 3 . The mean $V_{\text {rot }}(\mathrm{obs}) / \sigma$ ratio of the remnants is 0.36 , increasing to $V_{\text {rot }} / \sigma=0.46$ when we apply the statistical inclination correction to the rotational velocity. The individual progenitors of the binary systems have a mean rotational velocity of 59 (with a standard deviation of 38) $\mathrm{km} \mathrm{s}^{-1}$ or 105 (with a standard deviation of 96) $\mathrm{km} \mathrm{s}^{-1}$ when the inclination effects are corrected from the ellipticity of each progenitor. The corresponding observed and inclination-corrected $V_{\text {rot }} / \sigma$ ratios of the progenitors are 0.42 and 0.76 . The somewhat lower $V_{\text {rot }} / \sigma$ ratio of the remnants can only be attributed to their higher (than the progenitors) value of $\sigma$, since the difference between the pre- and post- coalescence rotational velocity is insignificant. In these calculations we have not attempted to correct the central velocity dispersion for inclination effects.

\section{Origin Of The ULIRG Remnants}

We infer the initial conditions of the mergers that lead to ultraluminous IR activity by comparing the kinematic properties of ULIRG remnants with those predicted by simulations in the literature. According to various authors (e.g. Bendo \& Barnes 2000; Naab \& Burkert 2003) the remnant $V_{\text {rot }}(\mathrm{obs}) / \sigma$ ratio depends upon the initial mass ratio of the merging galaxies. In the gas-free, $\mathrm{N}$ body simulations of binary mergers performed by Naab \& Burkert (2003) for several mass ratios and orientations, the major mergers produced slowly rotating remnants. Those authors suggested that the $V_{\text {rot }}(\mathrm{obs}) / \sigma$ ratio is $\sim 0.2$ for $1: 1$ and $\sim 0.4$ for 2:1 merger remnants, while it reaches higher values $(0.8)$ when the remnants originate from minor (4:1) mergers. Our remnants agree best with a 1:1 and 2:1 merger origin, also agreeing with the directly measured progenitor mass ratios of $\mathrm{Pa}-$ per I and confirming that ULIRGs are representative of the most violent local mergers. The results are similar when comparing to those of Naab et al. (2006b), who have presented similar contours for gas-rich, dissipative simulations of $1: 1$ and $3: 1$ mass ratios.

Naab \& Burkert (2003), Burkert \& Naab (2005), and Naab et al. (2006b) have also shown a connection between the $V_{\text {rot }}($ obs $) / \sigma$ ratio and the remnant ellipticity at the effective radius for several progenitor mass ratios. We overplot our results with those of the merger simulations in Fig. 4, where our ULIRGs are given in triangles. Each panel corresponds to mergers of different progenitor mass ratios (denoted at the upper-left corner of each panel). In bold, solid, and dotted contours we show the $90 \%, 70 \%$, and $50 \%$ probability of finding a merger remnant of each category in the enclosed region. These contours correspond to models that do not include gas (Naab \& Burk- 
ert 2003). For the dissipative merger remnants of Naab et al. (2006b), the $90 \%$ and $50 \%$ probabilities are shown as dark- and light-gray shaded areas respectively. A comparison of the predicted with the measured remnant dynamics indicates that ULIRGs are more likely to be formed by major mergers (see Paper I). We also find that ULIRGs have a narrower mass-ratio origin than the merger remnants in Rothberg \& Joseph (2006b), who have carried out a similar analysis on a sample of sources that are selected visually to have perturbed morphologies (see also $§ 5.3$ ).

The addition of gas to the simulations of Naab \& Burkert (2003) did not significantly influence the position of remnants on the $V_{\text {rot }}(\mathrm{obs}) / \sigma$ vs. $\epsilon$ diagram (Burkert \& Naab 2005; Naab et al. $2006 \mathrm{~b})$. However, the addition of star formation may result in an increase of the theoretically predicted $V_{\text {rot }}(\mathrm{obs}) / \sigma$ ratio and ellipticity, since new stars will be created upon a dynamically-cold disk, formed by gas that is not consumed during the merger (Barnes 2002; Springel 2000). This possible increase would shift the probability contours to higher values on both axes further supporting our findings that ULIRGs are produced mainly by mergers of galaxies of comparable masses. On the other hand, the position of ULIRGs on this diagram could be shifted upwards along the $V_{\text {rot }} / \sigma$ axis due to aperture correction effects. The $V_{\text {rot }}$ values used are not necessarily measured from apertures where the rotation curve has reached its flat part (van Albada et al. 1985). However, the facts that $V_{\text {rot }}$ has been measured from apertures between 0.5 and $2 R_{\text {eff }}$ and that the ellipticity at the effective radius does not depend upon the apertures used to measure $V_{\text {rot }}$ are good indications that such corrections will probably not be sufficiently large to alter our conclusions.

\section{End Products Of ULIRGs}

\subsection{The Masses Of Ultraluminous Merger Remnants}

A direct way to investigate the nature of the end products of ultraluminous mergers is to calculate the mass $m$ of their remnants. According to Bender et al. (1992) the bulge mass is related to the stellar kinematics as

$$
m=c_{2} \sigma_{100}^{2} R_{\mathrm{eff}},
$$

where $\sigma_{100}$ is the projected central velocity dispersion in units of $100 \mathrm{~km} \mathrm{~s}^{-1}, R_{\text {eff }}$ is in $\mathrm{kpc}$, and $m$ is in $10^{10} M_{\odot}$. The geometrical factor $c_{2}$ depends on the distribution of matter in the galaxy. Following Tacconi et al. (2002), we adopt $c_{2}=1.4$, as appropriate for a constant $m / \mathrm{L}$ King model whose tidal-to-core radius ratio is 50 , midway between those of dwarf and giant ellipticals. By combining the above, the total dynamical mass is computed from

$$
m=4.7 \times 10^{5}\left(3 \sigma^{2}+{V_{\text {rot }}}^{2}\right) R_{\mathrm{eff}},
$$

where $\sigma$ and $V_{\text {rot }}$ are now in units of $\mathrm{km} \mathrm{s}^{-1} R_{\text {eff }}$ is in $\mathrm{kpc}$, and $m$ is in $M_{\odot}$.

We use the mean values of the stellar dispersion and inclination-corrected rotational velocity from $\S 3$. For the objects that do not have a $V_{\text {rot }}$ measurement (see Table 3 ), we use their dispersion in combination with the mean inclinationcorrected $V_{\text {rot }} / \sigma$ ratio $(0.46)$ to infer it. We find that the mean dynamical mass of the remnants is $8.91 \times 10^{10} M_{\odot}$ (in good agreement with Tacconi et al. 2002), suggesting that ultraluminous activity mainly originates from mergers of sub- $m_{*}$ galaxies, for $m_{*}=1.4 \times 10^{11} M_{\odot}$ (Genzel et al. 2001 and references therein). For different values of $m_{*}$ (e.g that of Bell et al. 2003 adapted to our cosmology, $\left.8.3 \times 10^{10} \quad M_{\odot}\right)$ the sub- $m_{*}$ characterization of ULIRGs may change to $\sim m_{*}$. Still, in the local Universe, the disk progenitors of ULIRGs do not need to be as massive as e.g. the Milky Way.

ULIRG masses derived from $\mathrm{H} \alpha$ emission-line dispersions are also sub- $\mathrm{m}_{*}$ (Colina et al. 2005). Tracing the ionized gas by the [FeII] emission lines that appear in our spectra, we find that while the mean dispersion of the gas is similar to that of the stars, in individual cases gas and stellar dispersions may significantly deviate due to gas outflows. Gas kinematics in these ULIRGs will be presented in a forthcoming paper (Tacconi et al. 2006 , in preparation).

The result that ULIRGs are sub- $m_{*}$ to $m_{*}$ galaxies does not contradict findings from imaging studies that ULIRGs have NIR luminosities greater than $L_{*}$ (e.g. Sanders et al. 2000; Colina et al. 2001; Veilleux et al. 2006). ULIRGs can simultaneously be sub- $m_{*}$ and $(1-2) \times L_{*}$ (in the $H$-band) mergers since they are selected at the peak of their starburst, at which time their luminosity-to-mass ratio rises (see also Tacconi et 
al. 2002; Rupke et al. 2002; 2005a; 2005b).

\subsection{ULIRGs And The Fundamental Plane Of Early-type Galaxies}

The $V_{\text {rot }} / \sigma$ ratios of the ULIRG remnants indicate that mergers of ultraluminous infrared output lead to random-motion-dominated systems with a non-negligible rotational component. To investigate what type of ellipticals ultraluminous IR mergers form, Genzel et al. (2001) and Tacconi et al. (2002) placed our initial sample of ULIRGs on the fundamental plane of early-type galaxies (Djorgovski \& Davis 1987; Dressler et al. 1987) that relates the velocity dispersion, effective radius and surface brightness of these sources. Genzel et al. (2001) and Tacconi et al. (2002) concluded that the remnants resemble moderate mass ellipticals (of stellar mass $\sim 10^{11} M_{\odot}$ ). Our new data increase the number of objects in the ULIRG luminosity range to be compared with early-type galaxies.

The $R_{\text {eff }}-\sigma$ projection of the plane that we construct from our data is shown in Fig. 5. Data for early-type galaxies are taken from Bender et al. (1992), Faber et al. (1997), and Pahre (1999). Giant boxy ellipticals (squares) occupy the upperright corner of the FP projection, while disky, moderate-mass ellipticals (circles) are located at the center. On the left panel of Fig. 5 we overplot the ULIRG remnants in triangles; 29 are from this study and 2 from Rothberg \& Joseph (2006a), UGC $5101^{5}$ and AM 2246-490 The location of ULIRGs on the plane agrees very well with that of moderate-mass ellipticals. On the right panel of Fig. 5, we show where remnants of other populations are located. Local LIRGs (filled diamonds) lie from the lower-left corner of the FP projection up to the locus of giant Es (data from Shier \& Fischer 1998, James et al. 1999, Rothberg \& Joseph 2006a, and Hinz \& Rieke $2006^{6}$. The position of ULIRGs on this FP projection is more tightly restricted than that of LIRGs; this result implies that ULIRGs have a narrower range of intrinsic

\footnotetext{
${ }^{5}$ The $H$ - and $K$-band effective radii of this source equal 0.27 $\mathrm{kpc}$ and $1.42 \mathrm{kpc}$ respectively (Scoville et al. 2000; Rothberg \& Joseph 2004).

${ }^{6}$ Whenever several velocity dispersion and effective radius measurements exist in the literature for sources that are not included in our sample, we use the average value of those measurements.
}

dynamical properties than LIRGs. Other visuallyselected merger remnants (i.e. sources with perturbed morphology but no IR excess; Rothberg \& Joseph 2006a) are shown as open-crossed diamonds. That these merger remnants lie closer than both the ULIRGs and the LIRGs to the locus of giant Es possibly reflects a different merger origin (see $\S 5.3$ ).

In the 3-dimensional view of the plane, ULIRGs are known to deviate from the position of earlytype galaxies along the surface brightness axis due to extinction and population effects (e.g., Genzel et al. 2001). Due to the strong starbursts ULIRGs undergo, they have a significant population of newly formed asymptotic-giant-branch stars, red supergiants and giants. Thus, their NIR surface brightnesses are higher than those of quiescent ellipticals (Pahre 1999; Veilleux et al. 2002). Prior to comparing ULIRGs and Es, the removal of light originating from starburst components is therefore instructive. Since a significant fraction of the starburst emission is nucleated, we opt to remove the central PSF (Veilleux et al. 2006) simultaneously removing any AGN-originating emission.

In the $H$-band, the nuclear PSF removal has already been performed by Surace \& Sanders (1999), Colina et al. (2001) and Veilleux et al. (2006) for most of the sources in our sample. For the sources taken from Scoville et al. (2000), we use the mean $H$-band ratio between the luminosity of the PSF-subtracted galaxy and the total luminosity, which equals 0.64 (Surace \& Sanders 1999; Colina et al. 2001; Veilleux et al. 2006). The total magnitudes ${ }^{7}$ of approximately half of the sources we use to construct the 3-dimensional view of the $K$-band fundamental plane are from Kim et al. (2002). For the remaining sources, the data are from Duc et al. (1997), Surace \& Sanders (1999), Rigopoulou et al. (1999), and Scoville et al. (2000). In the $K$ band, the value of the ratio between the luminosity of the PSF-subtracted galaxy and the total luminosity is 0.75 times that in the $H$ band (Surace \& Sanders 1999) i.e., 0.48. We compute the mean surface brightness (within the effective radius) of each galaxy, $\left\langle\mu_{\text {gal }}\right\rangle$, by adding $2.5 \log \left(2 \pi R_{\text {eff }}^{2}\right)$ mags (where $R_{\text {eff }}$ is in ") to its PSF-subtracted magnitude in both bands. In

\footnotetext{
${ }^{7}$ The total magnitude here includes flux integrated up to the galaxy's truncation radius.
} 
Table 2 we tabulate the $H$ and the $K$-band value of $<\mu_{\text {gal }}>$ for each source.

In Fig. 6 (upper panel) we show the 3-dimensional view of the fundamental plane in the $H$-band for the 21 remnants of Table 2 with a $<\mu_{\text {gal }}(H-$ band) $>$ measurement. For viewing clarity, we also plot in Fig. 6 (lower panel) the fundamental plane in one of the representations introduced in Pahre (1999). $H$-band data for (mainly cluster) Es are from Zibetti et al. (2002) and references therein. The ellipticals are plotted as open circles ${ }^{8}$ and the ULIRGs as triangles. In the $H$-band, the individual PSF subtraction brings ULIRGs close to the fundamental plane, indicating that ULIRG remnants will resemble Es once their on-going starbursts cease. That ULIRGs are on average somewhat brighter than Es is justified by the fact that a (diffuse) part of their starburst emission remains unremoved after the PSF subtraction. In Fig. 7 we show the $K$-band 3 -dimensional view and Pahre (1999) visualization of the plane for 25 remnants in this study with a $<\mu_{\text {gal }}(K-$ band $)>$ measurement and two sources from Rothberg \& Joseph (2006a). In the $K$-band, ULIRGs lie again close to moderate-mass Es, after the statistical PSF removal. Along the photometric axis of the plane, PSF-subtracted ULIRGs are also close to LIRGs and other merger remnants.

We have not attempted to correct the photometric data of any wavelength for extinction effects; the value of the usually-assumed equivalent screen correction (Scoville et al. 2000; Genzel et al. 2001; Tacconi et al. 2002) is uncertain and changes after removal of the nuclear PSF. The application of an extinction correction will make the surface brightness of ULIRGs brighter and require more fading for them to evolve onto the fundamental plane. No k-corrections have been applied since they are negligible for the majority of the sources in our sample.

\subsection{Discussion: The role of ULIRGs in the formation of local Es}

Our main conclusion from the fundamental plane analysis is that ULIRGs typically lie on the locus of local moderate-mass ellipticals whose

\footnotetext{
${ }^{8}$ To enhance the clarity of the diagram we have excluded the dwarf Es, since they do not fall on the plane of all other Es.
}

stellar mass is $10^{10}-10^{11} M_{\odot}$. To further investigate the type of elliptical galaxy that ULIRGs form at the highest rates, we compare the number densities of local ULIRG remnants and ellipticals as a function of their stellar velocity dispersion.

In Fig. 8, we plot the source number density per stellar dispersion in the range between 50 and $300 \mathrm{kms}^{-1}$; ULIRGs are plotted as a histogram and local SDSS ellipticals $(0.01<z<0.3$; Bernardi et al. 2003) as a solid line. For the ellipticals, $n(\sigma)$ is computed from the velocity dispersion function of Sheth et al. (2003). The $n(\sigma)$ distribution of ULIRGs is calculated by multiplying the $\%$ fraction of ULIRG remnants per $\sigma$ bin with the volume density of ULIRGs $\left(2.5 \times 10^{-7} \mathrm{Mpc}^{-3}\right.$, for our cosmology) from Sanders et al. (2003). To facilitate the comparison between the two $n(\sigma)$ distributions, we apply a normalization factor of $7 \times 10^{3}$ to the ULIRG histogram so that its mean has the same number density as that of the SDSS ellipticals. Physically, this normalization factor is related to the ratio between the time over which ellipticals have been formed and the lifetime of a single ultraluminous burst. Its value is only a rough estimate since it depends on the completeness of the $1 \mathrm{Jy}$ and the SDSS (sub-)samples and the fact that local ultraluminous mergers are not the only mechanism to produce elliptical galaxies.

The $n(\sigma)$ distribution of SDSS elliptical galaxies has a mean dispersion similar to that of ULIRGs, 157 (with a standard deviation of 54) $\mathrm{kms}^{-1}$. In other words, the descendants of ULIRGs will resemble the ellipticals that are most common in the local Universe. Local ULIRGs seem to form at highest rates sources of characteristic $\sigma$ between 130 and $160 \mathrm{~km} \mathrm{~s}^{-1}$. That local Es are mainly produced by mergers of spirals of specific luminosities (or masses) is also found in the recent simulations of Kaviraz et al. (2006).

At low dispersions, the ratio between $n(\sigma)$ and $n(\langle\sigma\rangle)$ seems to be lower for ULIRGs than for ellipticals. This deviation can probably be attributed to the fact that mergers of gas-rich galaxies below a certain mass threshold do not possess enough gas to undergo a ULIRG phase.

Deviations between the two distributions also exist at the high-dispersion end. For most bins at the high-dispersion end, the ULIRG distribution seems again underpopulated compared to that of SDSS ellipticals. We cannot formally rule out the 
possibility that the most massive ULIRGs have been missed due to sample incompleteness, small volume densities, or possibly shorter (than the average) burst timescales for the most massive sources. However, one source with high velocity dispersion, Mrk 273, has been observed in our sample (see also Hinz \& Rieke 2006). Even for Mrk 273, the total mass of the system (which also depends on its effective radius) is insufficient to classify it as a giant elliptical (of stellar mass $\left.>5 \times 10^{11} M_{\odot}\right)$. Moreover, the fundamental plane analysis for all ULIRGs in our sample seems to suggest that ULIRGs do not account for the formation of a significant fraction of the giant elliptical galaxies in the local Universe. To quantify this statement, we apply a KS test to the ULIRG remnants and the (literature-selected) giant Es we used to construct the fundamental plane, acknowledging the different space-volumes they occupy. We find that the probability that ULIRG remnants and the giant ellipticals originate from the same population is extremely small, $10^{-9}$ (see also Genzel et al. 2001); this probability indicates that despite the small number statistics, the confidence of this result is very high. For their morphologically selected remnant sample, Rothberg \& Joseph (2006a) find a higher, but still insignificant, probability of $10 \%$.

To further assess how typicially ULIRGs form giant Es, we used the mass function of local Es to estimate what fraction $f_{\text {giant }}$ of the sources with mass $>10^{10} M_{\odot}$ (mass threshold similar to that of ULIRGs) correspond to giant ellipticals (of stellar mass $\left.>5 \times 10^{11} M_{\odot}\right)$. Given that the fraction may significantly vary according to the adopted mass function, we used the best fit to the $K$ band mass function from various authors in the literature. Giant ellipticals comprise $18.7 \%$ of local Es with mass $>10^{10} M_{\odot}$ for the mass function that Bell et al. (2003) derived for Es in the Sloan Digital Sky (SDSS) and the Two Micron All Sky (2MASS) surveys assuming a diet ${ }^{9}$ Salpeter initial mass function (IMF). The fraction $f_{\text {giant }}$ is $20.1 \%$ for the 2MASS $K$-band luminosity function of local Es (Kochanek et al. 2001), which we convert to a mass function using $m / L=1.32 M_{\odot} / L_{\odot}($ Cole et al. 2001). This

\footnotetext{
${ }^{9}$ In this initial mass function, the number of low-mass stars is reduced leading to a total stellar mass reduced by $30 \%$.
}

$K$-band $m / L$ ratio is computed for both early- and late- type galaxies in the 2MASS and 2dF Galaxy Redshift Surveys, assuming a Salpeter IMF. An estimate of the $m / L$ ratio for local ellipticals only can be derived from the work of Cappellari et al. (2005a) using the mean $I$-band $m / L$ value and I-K color of the SAURON project sources. Combining the 2MASS $K$-band luminosity function with this early-type-galaxy $m / L$ value $\left(0.75 M_{\odot} / L_{\odot}\right)$ yields $f_{\text {giant }}=10.9 \%$. Although $f_{\text {giant }}$ is between $10 \%$ and $20 \%$, we do not observe any source of $m>5 \times 10^{11}$ $M_{\odot}$ in our sample of 29 ULIRG remnants. This fact indicates that local giant ellipticals cannot be accounted for, if assumed to originate only from local ultraluminous mergers.

One possible scenario for the formation of local giant Es is that most of these objects have formed at higher redshifts (e.g. Swinbank et al. 2006). This scenario is based on the fact that the more massive early-type galaxies are, the faster and the earlier their stellar populations have formed (Thomas et al. 2005; van Dokkum 2005 and references therein). It is reinforced by the argument that local giant Es are mainly located in clusters whereas ULIRGs lie in the field (Tacconi et al. 2002). Another likely scenario, which may hold simultaneously, is that other types of mergers (e.g. E-S or E-E ones) account for the formation of $\sim 60 \%$ of elliptical galaxies (Khochfar \& Burkert 2003; Kaviraz et al. 2006) at low redshift. E-S mergers enhance the star-formation activity already existing in one of the merging components and, for adequate amounts of gas, may appear as LIRGs. Gas-depleted E-E mergers (also known as "dry" mergers) are not easy to detect observationally (e.g, van Dokkum 2005; Bell et al. 2006) since they do not have other unambiguous signatures such as luminous/ultraluminous phase(s) beyond their perturbed morphologies; therefore, they can mainly be traced by visual identification. Dry merging is now believed to be a key element in the formation of local giant Es (Naab et al. 2006a) and is a likely explanation for the locations of some optically selected mergers in the fundamental plane (see $\S 5.2$ ).

Rothberg \& Joseph (2006a) recently compiled a sample of local merger remnants selected by visual inspection. Such a sample probably comprises merger remnants of all possible (S-S; E-S; E-E) categories. Rothberg \& Joseph (2006a) measured 
$\sigma$ from Ca II triplet (optical) spectroscopy for 38 remnants, of which, 10 were LIRGs and 2 were ULIRGs. They found that the hypothesis that the (U)LIRGs in their sample and giant ellipticals can be drawn from the same parent population has a probability of $10 \%$. Lake \& Dressler (1986) also obtained optical velocity dispersion measurements for 13 merger remnants. Of those, 10 have Infrared Astronomical Satellite (IRAS) fluxes; none is a ULIRG and only one source, AM 0921-225, is a LIRG $^{10}$. Rothberg \& Joseph (2006a) computed the probability that the remnants of Lake \& Dressler (1986) and giant Es originate from the same parent population and found it to be $5 \%$.

The conclusion from the comparison of the optically-selected remnants of Rothberg \& Joseph (2006a) and Lake \& Dressler (1986) with the IRselected remnants in our study (and those of Genzel et al. 2001 and Tacconi et al. 2002) is what is expected from a theoretical point of view: while mergers account for the formation of galaxies of various morphological classes and mass contents (e.g., Schweizer \& Seitzer 1992; Springel \& Hernquist 2005; Springel et al. 2005a), ultraluminous IR mergers seem to have a specific output (moderate-mass ellipticals) originating from their specific input (mostly sub-m ${ }_{*}$ spirals).

\subsection{Wavelength Dependence Of The Stel- lar Velocity Dispersion Measurement}

Systematic differences between the measured and the actual values of the host dispersion may arise when extracting $\sigma$ from different wavelengths, i.e. from the Ca II triplet in the optical (e.g. Rothberg \& Joseph 2006a) and the $\mathrm{CO}$ bandheads in the NIR. The host kinematics extracted from the NIR bandheads are often representative of young stellar populations. These populations could still be linked to the gas from which they formed (which is believed to settle into a disk earlier than the stars in the progenitor disks; Mihos \& Hernquist 1996), and have less perturbed orbits than the old stars. Host dispersions extracted from the $\mathrm{CO}$ bandheads could be systematically lower than those of the merg-

${ }^{10}$ We computed the IR luminosity of these sources with the formula of Sanders \& Mirabel (1996) using mid-infrared fluxes from Moshir et al. (1990), The Point Source Catalog (1988), and the NASA/IPAC Extragalactic Database ing bulges. However, the low $V_{\text {rot }} / \sigma$ ratio that we measure for our ULIRGs is a good indication that even the young stellar populations are significantly heated, and therefore, systematics originating from NIR dispersion measurement are unlikely to have a major effect on our conclusions.

In the case of the $\mathrm{Ca}$ triplet, systematic errors are mainly related to the presence of dust. Due to scattering of light from dust particles, photons originating from high-velocity stars in the center of the galaxy will be scattered into random lines of sight, biasing the LOS velocity distribution (Baes \& Dejonghe 2002). The effects of dust in the observed stellar kinematics depend upon the dust mass and extent (Baes \& Dejonghe 2002). Since ULIRGs are highly obscured systems, the stellar dispersions extracted from the Ca triplet may be systematically higher than their intrinsic values.

Such possible systematics have not been investigated in ULIRGs. Silge \& Gebhardt (2003) have attempted to quantify the discrepancy between dispersion estimates derived from the NIR and the optical regimes for local quiescent galaxies. While they found no significant difference for elliptical galaxies, the systematics were non-negligible in S0 lenticulars, with the largest difference in $\sigma(\sim 30$ $40 \%$ ) found in the most massive sources of their sample. The opposite conclusion is found from the velocity dispersion of ellipticals and Seyferttype AGNs in Oliva et al. (1995) and Oliva et al. (1999). The $\sigma$ values for the objects in their sample were systematically greater in the NIR than in the optical. The difference was large for type 1 Seyferts (up to a factor of 2) but low for type 2 Seyferts and ellipticals $(<10 \%)$. However, one of the uncertainties often underestimated in these comparisons is that the spectral resolution in the NIR was significantly lower than that in the optical. For Silge \& Gebhardt (2003), $R$ was 2300 in the $K$-band; for Oliva et al. $(1995 ; 1999)$ it was equal to 1900 and 2500 in the $H$ - and in the $K$-band respectively. To get a better estimate of the possible systematic errors in $\sigma$, we compare the results for LIRGs with a Ca II triplet $\sigma$ measurement (Rothberg \& Joseph 2006a) that also have $\mathrm{CO}$ observations of relatively high spectral resolution (e.g., equal or better than ours). These sources (NGC 1614, NGC 2623, NGC 4194, Arp 193, and IRAS 20551-4250) have been observed in the NIR by James et al. (1999), Gen- 
zel et al. (2001), Rothberg \& Joseph (2006a), and more recently, Hinz \& Rieke (2006). For most sources, the optical dispersions were higher than the NIR ones, but the systematic difference was small ( $7 \%$ on average). On the other hand, significant deviations (of a factor of 2) exist between the NIR results of James et al. (1999) and Hinz \& Rieke (2006) and the older (also NIR but lower-resolution) data of Shier \& Fischer (1998) for NGC 1614 and NGC 2623. It is therefore not clear whether the measurement of $\sigma$ from the different features would systematically yield different results for ULIRGs, and if yes, whether they would reflect real dynamic trends instead of measurement errors. Errors may also be easily introduced from the use of different stellar templates by various authors.

We investigate whether the use of the Ca II triplet for the measurement of $\sigma$ may affect our results on the formation of (giant) Es under the hypothesis that the optical $\sigma$ values can be indeed greater than the NIR ones due to population and extinction effects. We compute the maximum possible increase that can be applied to the dispersions of the sources in our sample, which is the maximum reported deviation (40\%) in the results of Silge \& Gebhardt (2003), corresponding to an addition of $\sim 60 \mathrm{~km} \mathrm{~s}^{-1}$ in $\sigma$ or a shift of 0.15 in the (logarithmic) horizontal axis of the fundamental plane. It is clear that this shift brings merger remnants closer to, but not into, the region populated by giant ellipticals. We also recompute the masses of the individual remnants in our sample after increasing their dispersion by $40 \%$. The average mass is now $1.69 \times 10^{11} M_{\odot}$, which is again close to $m_{*}$ and there is still no source more massive than $5 \times 10^{11} M_{\odot}$. Therefore, our main conclusions are insensitive to the wavelength dependence of the stellar velocity dispersion measurement. Furthermore, the $V_{\text {rot }}(\mathrm{obs}) / \sigma \operatorname{ratios}^{11}$ of S0 lenticulars are typically greater than those of ULIRGs; e.g, for the combined sample of Pizzela et al. (2005) and Cappellari et al. (2005b) the ratio is twice that of the remnants in our sample $(0.6)$ indicating that deviations of the order $30-40 \%$ between the $\mathrm{CO}$ and $\mathrm{Ca}$ dispersion measurement are probably high for ULIRGs.

\footnotetext{
${ }^{11}$ not corrected for inclination effects
}

\section{Black Holes in ULIRGs}

\subsection{A Picture Of The $M_{\mathrm{BH}^{-}} \sigma$ Relation Time Evolution}

The size of a black hole seems to be closely linked to the depth of the potential well in which it forms and grows (Ferrarese \& Merritt 2000). This is reflected, e.g., in the local $M_{\mathrm{BH}}-\sigma$ relation, the correlation between the black hole mass and the stellar dispersion in the bulge of the host galaxy (Ferrarese \& Merritt 2000; Gebhardt et al. 2000). Since the $M_{\mathrm{BH}}-\sigma$ relation is found for virialized bulges, it should be valid at the end of the merger process, when the bulge stellar kinematics have reached their final dynamical state and AGN winds and supernovae feedback have expelled the gas away from the nucleus, preventing further $\mathrm{BH}$ growth, terminating the starburst phase, and making the system resemble an elliptical galaxy. The merger remnants in recent simulations (Di Matteo et al. 2005; Springel et al. 2005; Robertson et al. 2005) are able to reproduce the $M_{\mathrm{BH}}-\sigma$ relation by subjecting a portion of their interstellar gas to accretion and feedback.

For ULIRGs however, the conversion of the host velocity dispersion into $M_{\mathrm{BH}}$ carries the uncertainty of applying the $M_{\mathrm{BH}}-\sigma$ relation to systems out of dynamical equilibrium. It is not yet known if, or to what extent, the $M_{\mathrm{BH}}-\sigma$ relation is valid between the first encounter and shortly ( $\lesssim 10^{8}$ yrs) after the final coalescence, when most of the ultraluminous infrared activity occurs.

To investigate whether (and under which conditions) merging disk galaxies fall on the $M_{\mathrm{BH}}-\sigma$ relation during interaction phases prior to coalescence, we ran gas-rich merger simulations (details presented in Naab et al. 2006b) that have already been discussed briefly in Paper I. To include the effects of a dissipative component we replaced $10 \%$ of the stellar mass in the initial disks with isothermal gas at a temperature of approximately $10^{4} \mathrm{~K}$. The initial scale length of the gas disk was equal to that of the stellar disk, $h$. Each galaxy had a stellar bulge with $1 / 3$ of the disk mass and was embedded in a pseudo-isothermal halo to guarantee a flat rotation curve at large radii. All galaxies approached each other on nearly parabolic orbits with a pericenter distance of two disk scale lengths. The spin orientations were both prograde and retrograde. The evolution of the 
stars and the gas was computed with the N-body smoothed-particle-hydrodynamics code VINE using an isothermal equation of state for the gas. For this study we analyzed mergers with 16 different initial disk orientations (geometries 1-16 of Naab \& Burkert 2003) and mass ratios 1:1 and 3:1.

We analyzed snapshots in the orbital plane approximately every half-mass rotation period of the more massive disk to follow the time evolution of the projected central stellar velocity dispersion and the gas accretion history onto the center of the system. At each snapshot, we calculated $M_{\mathrm{BH}}$ from the velocity dispersion assuming that the $M_{\mathrm{BH}}-\sigma$ relation is always valid. As in Paper I, we used the Tremaine et al. (2002) formula

$$
M_{B H}=1.35 \times 10^{8}[\sigma / 200]^{4.02} M_{\odot}
$$

where $\sigma$ is in units of $\mathrm{kms}^{-1}$, to calculate the black hole masses. To quantify the gas accretion, we computed the total gas mass $M_{\text {gas }}$ that has ever reached a radius of $0.1 \mathrm{~h}$ (i.e. twice the resolution element of the simulations) relative to the mass center of the system. By calculating the ratio between the quantities $M_{\mathrm{BH}}$ and $M_{\text {gas }}$ we derive the accretion efficiency $\epsilon$ that is needed to maintain the black hole mass on the Tremaine et al. (2002) formula at any time. We define accretion efficiency $\epsilon$ as the fraction of the gas that accretes onto the black hole from the gas accumulated into the nuclear region.

In Fig. 9 we show the evolution of the accretion efficiency (needed to keep the black hole on the $M_{\mathrm{BH}}-\sigma$ relation) as a function of time (left column) and nuclear separation (right column). Time is given in units of the half-mass rotation period, $T_{1 / 2}$, of the more massive progenitor disk. The nuclear separation is given in disk scale lengths of the more massive disk. The models are scale-free; scaling the parameters to e.g., those of the Milky Way corresponds to a unit time of 13 Myrs and a unit length of $3.5 \mathrm{kpc}$. Scaling them to the mean scale length and rotational velocity of all individual progenitors in our binary ULIRG sample yields a unit time of 13 Myrs and a unit length of $1.3 \mathrm{kpc}$. The efficiency is averaged over the 16 initial geometries for 1:1 and 3:1 mergers (upper and lower panels). The diagonally shaded area indicates the spread due to the varying initial disk geometries.

According to the left panels of Fig. 9, before nuclear coalescence, the estimated black hole mass and the gas accumulated at the center of the simulation already scale linearly. In other words, if we assume that the $M_{\mathrm{BH}}-\sigma$ relation is valid at any time during the merger, then we find that the accretion efficiency remains constant shortly after the first encounter (shown in vertical dashes) until relaxation. Vice-versa, if we assume that $\epsilon$ stays constant during the merger and require only that the $M_{\mathrm{BH}}-\sigma$ relation be valid at relaxation ${ }^{12}$, we find that the black hole mass can be computed from the $M_{\mathrm{BH}}-\sigma$ relation from midway of the first encounter through relaxation.

The implication of these simulations is that the $M_{\mathrm{BH}}-\sigma$ relation can be used to compute black hole masses in merging systems, from mid-way between first encounter and nuclear coalescence until the remnant becomes an elliptical galaxy, as long as $\epsilon$ remains constant. This is true for both equal(1:1) and unequal- (3:1) mass major mergers. A constant efficiency $\epsilon$ conceptually corresponds to subjecting a specific gas fraction to AGN feeding, an assumption also made for models that include star formation and ISM feedback (e.g. Springel et al. 2005). However, it is likely that on short timescales (e.g. during specific accretion events) the efficiency $\epsilon$ may vary significantly. Therefore individual black hole estimates calculated from the $M_{\mathrm{BH}}-\sigma$ relation carry this uncertainty.

In the right panels of Fig. 9, the "folding" of the efficiency $\epsilon$ at large nuclear separations is due to the fact that the galaxies move towards apogalacticon before they fall back together. Clearly, at these merger phases, the use of the $M_{\mathrm{BH}}-\sigma$ relation is misleading. For a constant $\epsilon$, the $M_{\mathrm{BH}}-\sigma$ relation begins to be valid beyond 5 disk scale lengths, which corresponds to a nuclear separation $\sim 7 \mathrm{kpc}$ for our ULIRGs (see Paper I). If the assumption of a constant $\epsilon$ holds, the black hole estimates for $\sim 2 / 3$ of the binary sources may be considered reasonable (but still lower limits on their final values). For the ULIRG remnants, computing $\mathrm{BH}$ masses from stellar dispersions seems also plausible. This is further supported by the fact that the kinematics of the latter are expected to have almost settled to their relaxation values (Mihos 2000). ${ }^{12}$ This assumption is reasonable since ULIRGs will eventually
form Es. 


\subsection{Black Hole Sizes and Accretion Rates}

We calculate the individual black hole masses of the sources in our sample using the Tremaine et al. (2002) formula and tabulate the results in Table 3. The mean black hole mass of the ULIRG remnants is $8.4 \times 10^{7} M_{\odot}$. Since our sources are still in an ultraluminous IR emission phase, gas and dust are still present in the nuclear region and will continue accreting onto the black hole. However, beyond coalescence, the timescales for further gas accretion onto the black hole are probably shorter than those of the pre-coalescence starbursts (e.g. Springel et al. 2005). Therefore, the black hole masses of the fully relaxed systems will be somewhat larger than those presented in Table 3. Simple gas content (typical gas mass of ULIRGs is $5 \times 10^{9} M_{\odot}$; Downes \& Solomon 1998) and accretion efficiency (0.01, see $\S 6.1)$ arguments indicate that the additional increase of the remnant $\mathrm{BH}$ masses will not exceed $10^{8} M_{\odot}$ by the time the ultraluminous activity ends. In this order-ofmagnitude calculation we have ignored the fact that only part of the gas reaches (or remains in) the center of the merging system, further reducing the upper limit on the final black hole mass.

We calculate the BH mass each source would have, if it were accreting at the Eddington rate

$$
L_{\mathrm{Edd}} / L_{\odot}=3.8 \times 10^{4} M_{\mathrm{Edd}} / M_{\odot} .
$$

To calculate the Eddington $\mathrm{BH}$ mass $M_{\text {Edd }}$, we assign to the Eddington luminosity $L_{\mathrm{Edd}}$ half of the luminosity emitted in the IR, given that some ULIRGs are largely starburst- while others are AGN- powered (Genzel et al. 1998; Rigopoulou et al. 1999; Veilleux et al. 1999; Joseph 1999; Sanders 1999). The Eddington efficiency $\eta_{\text {Edd }}$, the ratio of the Eddington $M_{\mathrm{BH}}$ estimate over the dynamical black hole mass, is given in Table 3 . The mean Eddington efficiency of the mergedULIRG sample is 0.89 , with a wide range of values. While statistically appropriate, the assumption that $50 \%$ of the IR luminosity originates from the AGN may make some sources appear as if accreting at super-Eddington rates (see Table 3). If we exclude these sources from the statistics and recalculate the average efficiency, we find it to be 0.39 .

The inferred accretion rates may be higher than in reality if an overestimated fraction of the IR emission is assigned to the AGN. This could occur if, for example, the starburst is still the dominant source of the IR luminosity after the merging nuclei coalesce. The relative strengths of the starburst $\left(\mathrm{L}_{\mathrm{SB}}\right)$ and the AGN ( $\left.\mathrm{L}_{\mathrm{AGN}}\right)$ luminosity at each merger phase are uncertain. However, the MIR ISO spectroscopic study of local ULIRGs by Genzel et al. (1998) and Rigopoulou et al. (1999) indicates that most ULIRGs are starburst dominated systems, implying that probably less than $50 \%$ of the IR luminosity should be assigned to the AGN. Veilleux et al. (2002) have found that the strength of nuclear continuum emission increases with decreasing nuclear separation. Therefore, the Eddington efficiencies of the progenitors presented in Paper I may be systematically overestimated compared to those of the remnants. Future papers presenting SPITZER MIR spectroscopy of local ULIRGs will indicate the appropriate luminosity fraction that needs to be assigned to the AGN for ULIRGs before and after nuclear coalescence.

\section{Conclusions}

We have acquired NIR spectroscopic, long-slit, data of 54 ULIRGs at a variety of merger phases to trace the evolution of their host dynamical properties. From the analysis of the stellar kinematics in 29 ULIRG remnants, we find that:

1. Indications of an increase of the stellar random motions exist as the merger advances. The mean stellar $\sigma$, as measured from the $\mathrm{CO}$ rovibrational bandheads, equals $142 \mathrm{~km} \mathrm{~s}^{-1}$ for the binary sources (of mean nuclear separation $9.4 \mathrm{kpc}$, including IRAS 00456-2904 and IRAS 09111-1007) and $161 \mathrm{~km} \mathrm{~s}^{-1}$ for the remnants (of nuclear separation $<1.5 \mathrm{kpc}$ ). This difference in the means of the pre- and post- coalescence distributions is marginally significant and requires more data to be accurately constrained. This increase of the stellar dispersion observed in ULIRGs corresponds to only a part of the dynamical heating that occurs during the merger, since the merger timescales are longer than those of the ultraluminous starburst.

2. The dynamical and structural properties of the remnants indicate that they originate 
from mergers mainly of $1: 1$ and $2: 1$ progenitor mass ratios. This confirms what we found in Paper I by directly measuring the masses of the individual progenitors of binary ULIRGs.

3. Ultraluminous mergers will mainly lead to the formation of moderate mass ellipticals (of stellar mass $\sim 10^{11} M_{\odot}$ ). Depending on the definition of $m_{*}$, ULIRGs are between sub- and $\sim m_{*}$. ULIRGs are located in a region of the fundamental plane of early-type galaxies different from that of local giant Es, indicating a different formation history for most of the latter. Local mass and dispersion functions support this argument; however, the current statistics cannot exclude the formation of few giant Es from ULIRGs.

4. We have performed simulations to investigate whether a black hole mass-host dispersion relation can be used to calculate the black hole masses of our ULIRGs. We find that already before nuclear coalescence, the mass of the gas that falls into the center of the merging system scales linearly with the black hole mass predicted by the $M_{\mathrm{BH}}-\sigma$ relation. However, this is only true (and conversely) if the efficiency of gas accretion onto the $\mathrm{BH}$ from its surroundings remains constant with time.

5. The black hole masses of the merged ULIRGs are of order $10^{7}-10^{8} M_{\odot}$ and their accretion rates are high (Eddington efficiencies often $>0.5)$. If the AGN luminosity output of a ULIRG nucleus increases with time, our accretion rates in pre-coalescence ULIRGs may be overestimated relative to those in post-coalescence ULIRGs.

We are grateful to N. Scoville for providing $H$ band NICMOS imaging data for seven sources and D. C. Kim for giving us prior-to-publication NICMOS photometric results for several sources. We would like to thank the ESO VLT staff for their support both in the service and visitor mode data acquisition. We acknowledge the detailed input of the anonymous referee that improved the clarity of the manuscript. S. Veilleux was supported in part by NASA grant GO-0987501. A. J. Baker acknowledges support from the National Radio Astronomy Observatory, which is operated by Associated Universities, Inc., under cooperative agreement with the National Science Foundation. 


\section{A. APPENDIX: Notes on individual sources}

IRAS 00091-0738: This source has two overlapping nuclei separated by $1.4 \mathrm{kpc}$. Archival Wide Field Planetary Camera 2 data of this source (PI Borne) also indicate an optical nuclear separation of $1.5 \mathrm{kpc}$.

IRAS 00456-2904: According to the imaging analysis of Kim et al. (2002) and Veilleux et al. (2006) this source is probably a binary system at projected nuclear separation of $22.2 \mathrm{kpc}$ (converted to our cosmology). The redshift of the NE nucleus is not spectroscopically confirmed but its ambiguous morphology indicates an interaction. The data presented in this paper are for the more luminous (late-type-host) SW nucleus.

IRAS 09111-1007: Duc et al. (1997) find that this source has two widely separated interacting nuclei; the ultraluminous IR component is the western source. The nuclear separation measured from our acquisition images equals $40.4 \mathrm{kpc}$.

IRAS 14348-1447: According to the NICMOS imaging of Scoville et al. (2000), this source has a projected nuclear separation of $6.0 \mathrm{kpc}$ (converted to our cosmology).

IRAS 14378-3651: Two components separated by $1.5 \mathrm{kpc}$ are visible in the acquisition image of this source.

IRAS 15250+3609: The two components of this source are separated by $0.8 \mathrm{kpc}$ (Scoville et al. 2000).

IRAS 17208-0014: Hinz \& Rieke (2006) measure the velocity dispersion of this source to be $125 \pm 28$ $\mathrm{km} \mathrm{s}^{-1}$.

IRAS 20551-4250: Rothberg \& Joseph (2006a) find a dispersion of $185 \pm 6 \mathrm{~km} \mathrm{~s}^{-1}$ for this source.

IRAS 23578-5307: Our acquisition image indicates the presence of two nuclei separated by $1.4 \mathrm{kpc}$.

Arp 220: This source has a projected nuclear separation of $0.3 \mathrm{kpc}$ (Scoville et al. 1998).

NGC 6240: The two nuclei of this source are separated by $1.4 \mathrm{kpc}$ and still show some discrete velocity patterns. The velocity dispersion of the system peaks between them; at the position of this peak the stellar kinematics may reflect localized motions of self-gravitating gas (Tecza et al. 2000; Genzel et al. 2001). For the velocity dispersion of this source we use the average of the values at two nuclei and at the internuclear peak $\left(229 \mathrm{~km} \mathrm{~s}^{-1}\right.$ ), which is close to the luminosity-averaged value, $225 \mathrm{~km} \mathrm{~s}^{-1}$ (Tecza et al. 2000).

Mrk 273: This system has two nuclei separated by $0.8 \mathrm{kpc}$ (Scoville et al. 2000). Hinz \& Rieke (2006) find a stellar velocity dispersion of $232 \pm 43 \mathrm{~km} \mathrm{~s}^{-1}$ for the north component of this source. 


\section{REFERENCES}

Baes, M. \& Dejonghe, H. 2002, MNRAS, 335, 441

Barnes, J. E. 1992, ApJ, 393, 484

Barnes, J. E, \& Hernquist, L., 1996, 471, 115

Barnes, J. E. 2002, MNRAS, 333, 481

Bell, E. F., McIntosh, D. H., Katz, N., Weinberg, M. D. 2003, ApJS, 149, 289

Bell, E. F. et al. 2006, ApJ, 640, 241

Bender, R. 1990, A\&A, 229, 441

Bender, R., Burstein, D., \& Faber, S. M. 1992, ApJ, 399, 462

Bendo, G. J., \& Barnes, J. E. 2000, MNRAS, 316, 315

Bernardi, M. et al. 2003, AJ, 125, 1817

Bertin, E., \& Arnouts, S. 1996, A\&AS, 117, 393

Bryant, P. M., \& Scoville, N. Z. 1999, AJ, 117, 2632

Burkert, A. \& Naab, T. 2005, MNRAS, 363, 597

Canalizo, G., \& Stockton, A. 2001, ApJ, 555, 719

Cappellari, M., et al. 2005a, in press, astro$\mathrm{ph} / 0505042$

Cappellari, M., et al. 2005b, in press, astro$\mathrm{ph} / 0509470$

Cole, S., et al. 2001, MNRAS, 326, 255

Colina, L, et al. 2001, ApJ, 563, 546

Colina, L., Arribas, S., Monreal-Ibero, A. 2005, ApJ, 621, 725

Collin, S., Kawaguchi, T., Peterson, B., \& Vestergaard, M. 2006, A\&A accepted, astro$\mathrm{ph} / 0603460$

Dasyra, K. M., Tacconi, L. J., Davies, R.I., Lutz, D., Genzel, R., Naab, T., Burkert, A., Veilleux, S. \& Sanders, D. 2005, ApJ, 638, 745

Di Matteo, T., Springel, V., \& Hernquist, L. 2005, Nature, 433, 604

Djorgovski, S., \& Davis, M. 1987, ApJ, 313, 59
Duc P.-A., Mirabel, I.F., \& Maza, J. 1997, A\&AS, 124,533

Downes, D., \& Solomon, P.M. 1998, ApJ, 507, 615

Dressler, A., Lynden-Bell, D., Burstein, D., Davies, R. L., Faber, S. M., Terlevich, R., \& Wegner, G. 1987, ApJ, 313, 42

Dunlop, J. S, McLure, R. J., Kukula, M. J., Baum, S. A., O'Dea, C. P., \& Hughes, D. H. 2003, MNRAS, 340, 1095

Faber, S. M., et al. 1997, AJ, 114, 1771

Ferrarese, L., \& Merritt, D. 2000, ApJ, 539, L9

Gao, Y., \& Solomon, P. M. 1999, ApJ, 512, L99

Gebhardt, K. et al. 2000, ApJ, 543, L5

Genzel, R., et al. 1998, ApJ, 498, 579

Genzel, R., Tacconi, L. J., Rigopoulou, D., Lutz, D., \& Tecza, M. 2001, ApJ, 563, 527

Hernquist, L. 1993, ApJ, 409, 548

Hinz, J. L., \& Rieke, G. H., 2006, astro$\mathrm{ph} / 0604286$

James, P., Bate, C., Wells, M., Wright, G., \& Doyon, R. 1999, MNRAS, 309, 585

Joint Iras Science Working Group, 1988, Infrared Astronomical Satellite Catalogs, The Point Source Catalog v. 2.0, IRASP, C2, 0000

Joseph, R. D. 1999, Ap\&SS, 266, 321

Kauffmann, G.; White, S. D. M. 1993, MNRAS, 261,921

Kaviraz, S., Devriendt, J. E. G., Ferreras, I., Yi, S. K., \& Silk, J. 2006, MNRAS, submitted, astro$\mathrm{ph} / 0602347$

Khochfar, S., Burkert, A. 2003ApJ, 597L, 117

Kim, D.-C., \& Sanders, D. B. 1998, ApJS, 119, 41

Kim, D.-C., Veilleux, S., \& Sanders, D. B. 2002, ApJS, 143, 277

Kochanek, C. S., et al. 2001, ApJ, 560, 566

Kormendy, J., Sanders, D. B. 1992, ApJ, 390L, 53 
Lake, G., \& Dressler, A. 1986, ApJ, 310, 605

Le Fèvre, O., et al. 2000, MNRAS, 311, 565

Lonsdale, C. J., Farrah, D., \& Smith, H. E., 2006, in "Astrophysics Update 2 - topical and timely reviews on astronomy and astrophysics", ed. J. W. Mason, Springer/Praxis books, astro$\mathrm{ph} / 0603031$

Lucy, L. B. 1974, AJ, 79, 745

Lutz, D., Veilleux, S., \& Genzel, R. 1999, ApJ, $517 \mathrm{~L}, 13$

Mihos, J. C., \& Hernquist, L. 1996, ApJ, 464, 641

Mihos, J. C., \& Bothun, G. D. 1998, ApJ, 500, 619

Mihos, J. C. 1999, Ap\&SS, 266, 195

Mihos, J. C. 2000, ASPC, 197, 275

Moorwood, A. F. M., et al. 1998, Messenger, 94, 7

Moshir, M. et al., 1990, Infrared Astronomical Satellite Catalogs, The Faint Source Catalog, v 2.0, IRASF, C, 0000

Murphy T. W., Soifer, B. T., Matthews, K., \& Armus, L. 2001, ApJ, 559, 201

Naab, T., \& Burkert, A. 2003, ApJ, 597, 893

Naab, T., Khochfar, S., \& Burkert, A. 2006, ApJ, 636, L81

Naab, T., Jesseit, R., \& Burkert, A. 2006, astro$\mathrm{ph} / 0605155$

Oliva, E., Origlia, L., Kotilainen, J. K., \& Moorwood, A. F. M. 1995, A\&A, 301, 55 O

Oliva, E., Origlia, L., Maiolino, R., \& Moorwood, A. F. M. 1999, A\&A, 350, 9

Pahre, M. A. 1999, ApJS, 124, 127

Pizzella, A., Corsini, E. M., Dalla Bonta, E., Sarzi, M., Coccato, L., Bertola, F. 2005, ApJ, 631, 785

Richardson, W. H. 1972, OSAJ, 62, 55

Rigopoulou, D., Spoon, H. W. W., Genzel, R., Lutz, D., Moorwood, A. F. M., \& Tran, Q. D. 1999, AJ, 118, 2625
Robertson, B., Hernquist, L., Cox, T. J., Di Matteo, T., Hopkins, P. F., Martini, P., Springel, V. 2005, ApJ, submitted, astro-ph/0506038

Rothberg, B., \& Joseph, R. D. 2004, AJ, 128, 2098

Rothberg, B., \& Joseph, R. D. 2006, AJ, 131, 185

Rothberg, B., \& Joseph, R. D. 2006, AJ, in press, astro-ph/0604493

Rupke, D. S., Veilleux, S., \& Sanders, D. B. 2002, ApJ, 570, 588

Rupke, D. S., Veilleux, S., \& Sanders, D. B. 2005, ApJS, 160, 115

Rupke, D. S., Veilleux, S., \& Sanders, D. B. 2005, ApJ, 632, 751

Sanders, D. B., \& Mirabel, I. F. 1996, ARA\&A, 34,749

Sanders, D. B. 1999, Ap\&SS, 266, 331

Sanders, D. B., Kim, D. C., Mazzarella, J. M., Surace, J. A., \& Jensen, J. B. 2000, ASPC, 197, 295

Sanders, D. B., Mazzarella, J. M., Kim, D.-C., Surace, J. A., Soifer, B. T. 2003 AJ, 126, 1607

Schmidt, M. \& Green, R. 1983, ApJ, 269, 352

Schweizer, F., \& Seitzer, P. 1992, AJ, 104, 1039

Scoville, N. Z., et al. 1998, ApJ, 492, L107

Scoville, N. Z., Evans, A. S., Thompson, R., Rieke, M., Hines, D. C., Low, F. J., Dinshaw, N., Surace, J. A., \& Armus, L. 2000 AJ, 119, 991

Sheth, R. K., et al. (2003), ApJ, 594, 225

Shier, L. M., \& Fischer, J. 1998, ApJ, 497,163

Silge, J., \& Gebhardt, K. 2003, AJ, 125, 2809

Springel, V. 2000, MNRAS, 312, 859

Springel, V., Di Matteo, T., \& Hernquist, L. 2005, MNRAS, 361, 776

Springel, V., Di Matteo, T., \& Hernquist, L. 2005 ApJ, 620, L79

Springel, V., \& Hernquist, L. 2005 ApJ, 622, L9 
Stanford, S. A., \& Bushouse, H. A. 1991, ApJ, 371,92

Surace, J. A., \& Sanders, D. B. 1999, ApJ, 512, 162

Swinbank, M., Chapman, S., Smail, I., Lindner, C., Borys, C., Blain, A., Ivison, R., \& Lewis, G., MNRAS, in press, astro-ph/0606372

Tacconi, L. J., Genzel, R., Lutz, D., Rigopoulou, D., Baker, A. J., Iserlohe, C., \& Tecza, M. 2002, ApJ, 580, 73

Tecza, M., Genzel, R., Tacconi, L. J., Anders, S., Tacconi-Garman, L. E., \& Thatte, N. 2000, ApJ, 537, 178

Thomas, D., Maraston, C., Bender, R., de Oliveira, C. M. 2005, ApJ, 621, 673

Toomre, A. 1977, in Evolution of Galaxies and Stellar Populations, ed. B. M. Tinsley \& R. B. Larson (New Haven: Yale University Observatory), 401

Tremaine, S., et al. 2002 ApJ, 574, 740

van Albada, T. S., Bahcall, J. N., Begeman, K., \& Sancisi, R. 1985, ApJ, 295, 305

van Dokkum, P. G. 2005, AJ, 130, 2647

Veilleux, S., Kim, D.-C., \& Sanders, D. B. 1999, ApJ, 522, 113

Veilleux, S., Kim, D.-C., \& Sanders, D. B. 2002, ApJS, 143, 315

Veilleux, S., et al. 2006, ApJ, 643, 707

Wright, G. S,., James, P. A., Joseph, R.D., \& McLean, I. S. 1990, Nature, 344, 417

Zibetti, S., Gavazzi, G., Scodeggio, M., Franzetti, P., \& Boselli, A. 2002, ApJ, 579, 261

This 2-column preprint was prepared with the AAS LATEX macros v5.2. 
TABLE 1

SOURCE LisT

\begin{tabular}{|c|c|c|c|c|c|c|c|}
\hline $\begin{array}{l}\text { Galaxy } \\
\text { (IRAS) }\end{array}$ & $\begin{array}{c}\mathrm{RA} \\
(2000)\end{array}$ & $\begin{array}{c}\text { Dec } \\
(2000)\end{array}$ & $z$ & $\log \left(L_{\mathrm{IR}} / \mathrm{L}_{\odot}\right)$ & $\begin{array}{l}\text { slit P.A. } \\
\left({ }^{\circ}\right)\end{array}$ & $t_{\text {integration }}$ & $\begin{array}{l}\text { Merger phase } \\
\text { classification }\end{array}$ \\
\hline $00091-0738$ & $00: 11: 43.3$ & $-07: 22: 08$ & 0.118 & 12.19 & 17,106 & 60,60 & $\mathrm{R}$ \\
\hline $00199-7426 \mathrm{~b}$ & $00: 22: 07.0$ & $-74: 09: 42$ & 0.096 & 12.23 & $-15,75,74$ & $60,60,60$ & $\mathrm{U}$ \\
\hline $00262+4251^{\mathrm{c}}$ & $00: 28: 54.0$ & $+43: 08: 18$ & 0.0927 & 12.02 & 45,0 & 20,60 & $\mathrm{R}$ \\
\hline $00397-1312$ & $00: 42: 15.5$ & $-12: 56: 04$ & 0.262 & 12.90 & $-1,89$ & 120,120 & $\mathrm{R}$ \\
\hline $00456-2904^{\mathrm{c}}$ & $00: 48: 06.8$ & $-28: 48: 19$ & 0.110 & 12.12 & 30 & 40 & B \\
\hline F01004-2237 & $01: 02: 49.9$ & $-22: 21: 57$ & 0.118 & 12.24 & $-1,89$ & 60,60 & $\mathrm{R}$ \\
\hline 01166-0844 b & $01: 19: 07.6$ & $-08: 29: 10$ & 0.118 & 12.03 & $-60,29,29$ & $60,60,60$ & B \\
\hline $01388-4618^{c}$ & $01: 40: 55.9$ & $-46: 02: 53$ & 0.090 & 12.03 & 0,90 & 40,40 & $\mathrm{R}$ \\
\hline $01572+0009\left(\right.$ Mrk 1014) ${ }^{\mathrm{c}}$ & $01: 59: 50.2$ & $+00: 23: 41$ & 0.163 & 12.53 & $20,-70$ & 80,60 & $\mathrm{R}$ \\
\hline F02021-2103 & $02: 04: 27.3$ & $-20: 49: 41$ & 0.116 & 12.01 & 53,142 & 60,60 & $\mathrm{R}$ \\
\hline $02364-4751 \mathrm{~b}$ & $02: 38: 13.1$ & $-47: 38: 11$ & 0.098 & 12.10 & 0,90 & 60,50 & B \\
\hline $04103-2838$ & $04: 12: 19.5$ & $-28: 30: 24$ & 0.117 & 12.55 & 89 & 60 & $\mathrm{R}$ \\
\hline $04313-1649$ & $04: 33: 37.1$ & $-16: 43: 32$ & 0.268 & 12.55 & $-1,89$ & 120,120 & $\mathrm{R}$ \\
\hline $05189-2524$ & $05: 21: 01$ & $-25: 21: 46$ & 0.043 & 12.09 & $-1,89$ & 200,160 & $\mathrm{R}$ \\
\hline 06035-7102 b & $06: 02: 54.0$ & $-71: 03: 10$ & 0.0795 & 12.12 & $65,153,153$ & $60,50,60$ & B \\
\hline $09039+0503$ & $09: 06: 34.2$ & $+04: 51: 25$ & 0.125 & 12.07 & $-1,89$ & 60,60 & $\mathrm{R}$ \\
\hline $09111-1007$ & $09: 13: 38.8$ & $-10: 19: 20$ & 0.054 & 11.95 & 34,124 & 120,60 & B \\
\hline $10190+1322 \mathrm{~b}$ & $10: 21: 42$ & $13: 07: 01$ & 0.077 & 12.00 & $64,149,149$ & $40,40,40$ & B \\
\hline $10565+2448 \mathrm{~b}$ & $10: 59: 18.1$ & $24: 32: 34$ & 0.0431 & 12.02 & $-66,24$ & 40,40 & B \\
\hline $11095-0238 \mathrm{~b}$ & $11: 12: 03$ & $-02: 54: 18$ & 0.106 & 12.20 & 39,129 & 120,120 & $\mathrm{~B}$ \\
\hline $11223-1244$ & $11: 24: 50$ & $-13: 01: 13$ & 0.199 & 12.59 & $-1,89$ & 80,80 & $\mathrm{U}$ \\
\hline $12071-0444$ b & $12: 09: 45.1$ & $-05: 01: 14$ & 0.128 & 12.35 & $-1,89$ & 60,60 & B \\
\hline $12112+0305 \mathrm{~b}$ & $12: 13: 47$ & $02: 48: 34$ & 0.073 & 12.28 & 37,99 & $60,60,40$ & B \\
\hline $12540+5708\left(\right.$ Mrk 231) ${ }^{c}$ & $12: 56: 14.2$ & $-56: 52: 25$ & 0.042 & 12.50 & $10,-30,-80$ & $40,40,40$ & $\mathrm{R}$ \\
\hline $13335-2612 \mathrm{~b}$ & $13: 36: 22$ & $-26: 27: 31$ & 0.125 & 12.06 & -5 & 100 & B \\
\hline $13428+5608\left(\right.$ Mrk 273) ${ }^{\mathrm{c}}$ & $13: 44: 42.1$ & $-55: 53: 13$ & 0.037 & 12.13 & 15,95 & 40,40 & $\mathrm{R}$ \\
\hline $13451+1232 \mathrm{~b}$ & $13: 47: 33$ & $12: 17: 23$ & 0.122 & 12.28 & 104,13 & 80,120 & B \\
\hline $14070+0525$ & $14: 09: 31.3$ & $+05: 11: 31$ & 0.264 & 12.76 & $-1,89$ & 120,120 & $\mathrm{R}$ \\
\hline $14348-1447^{\mathrm{c}}$ & $14: 37: 38.3$ & $-15: 00: 23$ & 0.0823 & 12.3 & 30 & 240 & $\mathrm{~B}$ \\
\hline $14378-3651^{\mathrm{c}}$ & $14: 40: 58.9$ & $-37: 04: 33$ & 0.068 & 12.24 & -45 & 80 & $\mathrm{R}$ \\
\hline $15130-1958$ & $15: 15: 55.2$ & $-20: 09: 17$ & 0.109 & 12.09 & $-171,-81$ & 80,110 & $\mathrm{R}$ \\
\hline $15250+3609^{c}$ & $15: 26: 59.4$ & $-35: 58: 38$ & 0.055 & 11.99 & $45,-45$ & 40,40 & $\mathrm{R}$ \\
\hline $15327+2340(\operatorname{Arp} 220)^{c}$ & $15: 34: 57.1$ & $23: 30: 11$ & 0.0181 & 12.1 & $52,-91$ & 120,120 & $\mathrm{R}$ \\
\hline $15462-0450$ & $15: 48: 56.8$ & $-04: 59: 34$ & 0.100 & 12.16 & $179,-91$ & 180,160 & $\mathrm{R}$ \\
\hline $16156+0146 \mathrm{~b}$ & $16: 18: 08$ & $01: 39: 21$ & 0.132 & 12.04 & $-50,-51,40,40$ & $60,60,60,60$ & B \\
\hline $16300+1558 \mathrm{~b}$ & $16: 32: 20$ & $15: 51: 49$ & 0.242 & 12.63 & $-1,89$ & 150,90 & B \\
\hline $16504+0228(\text { NGC } 6240)^{\mathrm{c}}$ & $16: 52: 58.9$ & $02: 24: 03$ & 0.0245 & 11.8 & $-158,-31$ & 20,20 & $\mathrm{R}$ \\
\hline $17208-0014^{\mathrm{c}}$ & $17: 23: 21.9$ & $-00: 17: 00$ & 0.0428 & 12.33 & 90,120 & 30,30 & $\mathrm{R}$ \\
\hline $19254-7245 \mathrm{~b}$ & $19: 31: 21.4$ & $-72: 39: 18$ & 0.0617 & 12.00 & $-13,77$ & 60,60 & B \\
\hline $20046-0623$ b & $20: 07: 19.3$ & $-06: 14: 26$ & 0.0844 & 11.97 & 69,159 & 60,60 & B \\
\hline $20087-0308^{c}$ & $20: 11: 23.2$ & $-02: 59: 54$ & 0.106 & 12.40 & $-45,45$ & 40,40 & $\mathrm{R}$ \\
\hline $20414-1651$ & $20: 44: 18.2$ & $-16: 40: 16$ & 0.087 & 12.26 & 54,144 & 100,80 & $\mathrm{R}$ \\
\hline $20551-4250^{\mathrm{c}}$ & $20: 58: 26.9$ & $-42: 39: 06$ & 0.0428 & 11.98 & $-45,45$ & 60,60 & $\mathrm{R}$ \\
\hline $21130-4446 \mathrm{~b}$ & $21: 16: 18.5$ & $-44: 33: 38$ & 0.0926 & 12.02 & 33 & 40 & B \\
\hline $21208-0519^{b}$ & $21: 23: 29$ & $-05: 06: 59$ & 0.13 & 12.01 & $-164,109,109$ & $60,60,60$ & B \\
\hline $21219-1757$ & $21: 24: 41.6$ & $-17: 44: 46$ & 0.112 & 12.06 & $-1,89$ & 50,40 & $\mathrm{R}$ \\
\hline $21329-2346^{\mathrm{b}}$ & $21: 35: 45$ & $-23: 32: 36$ & 0.125 & 12.09 & 31 & 60 & B \\
\hline $21504-0628$ b & $21: 53: 05.5$ & $-06: 14: 50$ & 0.078 & 11.92 & $\begin{array}{l}-39,59 \\
-76\end{array}$ & $\begin{array}{c}60,60 \\
60,60-60\end{array}$ & $\mathrm{R}$ \\
\hline $22491-1808 \mathrm{~b}$ & $22: 51: 49.2$ & $-17: 52: 23$ & 0.0778 & 12.09 & $-76,13,13$ & $60,60,60$ & B \\
\hline $\begin{array}{l}23128-5919^{b} \\
23230-6926\end{array}$ & $\begin{array}{l}23: 15: 46.8 \\
23: 26: 03.6\end{array}$ & $\begin{array}{l}-59: 03: 15 \\
-69: 10: 19\end{array}$ & $\begin{array}{l}0.045 \\
0.106\end{array}$ & $\begin{array}{l}11.96 \\
12.17\end{array}$ & $\begin{array}{c}-5,84,84 \\
-1,89\end{array}$ & $\begin{array}{c}40,40,40 \\
60,60\end{array}$ & $\begin{array}{l}\mathrm{B} \\
\mathrm{R}\end{array}$ \\
\hline $23234+0946 \mathrm{~b}$ & $23: 25: 56.2$ & $10: 02: 50$ & 0.128 & 12.05 & $-64,25$ & 60,60 & B \\
\hline $23365+3604^{c}$ & $23: 39: 01.3$ & $+36: 21: 10$ & 0.0645 & 12.09 & $45,-30$ & 15,40 & $\mathrm{R}$ \\
\hline $23578-5307^{\mathrm{c}}$ & $00: 00: 23.6$ & $-52: 50: 28$ & 0.125 & 12.10 & 107,14 & 60,40 & $\mathrm{R}$ \\
\hline
\end{tabular}

Note.- The coordinates, the redshift, the infrared luminosity, as well as the position angles and respective integration time for all our sources are presented in this Table. The data are presented in this paper unless otherwise noted.

a Pre- or post- coalescence classification, according to the scheme described in $\S 2.2$. "B" refers to a binary (pre-coalescence) source. " $\mathrm{R}$ " refers to a remnant (in post-coalescence). Remnants may also have two nuclei, separated by no more than $1.5 \mathrm{kpc}$. "U" indicates that the classification is uncertain.

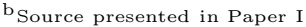

${ }^{\mathrm{c}}$ Source presented in Genzel et al. (2001) and Tacconi et al. (2002). 
TABLE 2

DERIVED STRUCTURAL AND PHOTOMETRIC PARAMETERS

\begin{tabular}{|c|c|c|c|c|c|c|}
\hline $\begin{array}{l}\text { Galaxy } \\
\text { (IRAS) }\end{array}$ & $\begin{array}{c}R_{\mathrm{eff}}(H \text { band }) \\
\quad(\mathrm{kpc})\end{array}$ & ellipticity & $\begin{array}{l}\phi_{\alpha} \\
\left(^{\circ}\right)\end{array}$ & $\begin{array}{c}R_{\mathrm{eff}}(K \text { band }) \\
(\mathrm{kpc})\end{array}$ & $\begin{array}{c}\mu_{\text {gal }}(H \text { band }) \\
\operatorname{mag}\left({ }^{\prime \prime}\right)^{-2}\end{array}$ & $\begin{array}{c}\mu_{\text {gal }}(K \text { band }) \\
\text { mag }\left({ }^{\prime \prime}\right)^{-2}\end{array}$ \\
\hline $00091-0738$ & $2.47( \pm 0.21)$ & 0.220 & 15 & $\ldots$ & $\ldots$ & $16.8^{\mathrm{h}}$ \\
\hline $00262+4251^{\mathrm{a}}$ & $\ldots$ & $\ldots$ & $\ldots$ & $3.4( \pm 1.0)^{\mathrm{e}}$ & $\ldots$ & $\ldots$ \\
\hline $00397-1312$ b & $2.04( \pm 0.76)$ & 0.35 & -25 & $\ldots$ & 16.4 & $15.2^{\mathrm{h}}$ \\
\hline F01004-2237 b & $0.40( \pm 0.07)$ & 0.02 & 26 & $\ldots$ & 14.3 & $12.9^{\mathrm{k}}$ \\
\hline $01388-4618$ & $1.62( \pm 0.03)$ & 0.074 & 55 & $\ldots$ & $\ldots$ & $14.7^{1, h}$ \\
\hline $01572+0009$ & $1.31( \pm 0.10)$ & 0.140 & 81 & $3.16( \pm 1.42)^{\mathrm{c}}$ & $14.6^{\mathrm{d}}$ & $13.7 \mathrm{k}$ \\
\hline F02021-2103 b & $5.38( \pm 3.95)$ & 0.34 & 1 & $\ldots$ & 17.1 & $17.2^{\mathrm{h}}$ \\
\hline $04103-2838^{\mathrm{b}}$ & $1.61( \pm 0.12)$ & 0.19 & -80 & $\ldots$ & 15.6 & $15.0^{\mathrm{h}}$ \\
\hline $04313-1649 \mathrm{~b}$ & $4.04( \pm 0.89)$ & 0.26 & 86 & $5.7^{\mathrm{f}}$ & 18.5 & $18.0^{\mathrm{h}}$ \\
\hline $05189-2524^{\mathrm{b}}$ & $0.57( \pm 0.08)$ & 0.06 & 77 & $0.79( \pm 0.02)^{\mathrm{c}}$ & 13.6 & $13.5^{\mathrm{k}}$ \\
\hline $09039+0503 \mathrm{~b}$ & $1.62( \pm 0.90)$ & 0.15 & -18 & $\ldots$ & 16.2 & $15.5^{\mathrm{h}}$ \\
\hline $11223-1244$ & $3.83( \pm 0.38)$ & 0.101 & 70 & $\ldots$ & $\ldots$ & $16.1^{\mathrm{h}}$ \\
\hline $12540+5708^{b}$ & $1.40( \pm 0.21)$ & 0.08 & -11 & $0.2^{\mathrm{e}}$ & 13.4 & $13.2^{\mathrm{k}}$ \\
\hline $13428+5608^{\mathrm{c}}$ & $1.03( \pm 0.29)$ & 0.498 & -77 & $1.15( \pm 0.24)^{\mathrm{c}}$ & $14.5^{\mathrm{d}, h}$ & $14.1 \mathrm{~d}, h$ \\
\hline $14070+0525 \mathrm{~b}$ & $3.62( \pm 0.53)$ & 0.17 & -19 & $\ldots$ & 17.2 & $16.2^{\mathrm{h}}$ \\
\hline $14378-3651$ & $0.36( \pm 0.02)$ & 0.031 & 20 & $0.67^{\mathrm{e}}$ & $14.6^{\mathrm{i}}$ & $13.4^{\mathrm{j}, h}$ \\
\hline $15130-1958^{\mathrm{b}}$ & $1.62( \pm 0.27)$ & 0.23 & -88 & $\ldots$ & 16.1 & $15.0^{\mathrm{h}}$ \\
\hline $15250+3609^{\mathrm{c}}$ & $2.10( \pm 0.09)$ & 0.207 & -72 & $1.49( \pm 0.12)^{\mathrm{c}}$ & $16.9^{\mathrm{d}, h}$ & $16.5^{\mathrm{d}, h}$ \\
\hline $15462-0450^{\mathrm{b}}$ & $5.57( \pm 1.38)$ & 0.048 & 62 & $1.6^{f}$ & 17.5 & $16.2^{\mathrm{h}}$ \\
\hline $17208-0014$ & $1.69( \pm 0.08)$ & 0.196 & 54 & $1.63( \pm 0.07)^{\mathrm{c}}$ & $15.9^{\mathrm{d}, h}$ & $15.6^{\mathrm{d}, h}$ \\
\hline $20087-0308$ & $1.87( \pm 0.22)$ & 0.324 & -86 & $\ldots$ & $\ldots$ & $15.2^{\mathrm{j}, h}$ \\
\hline $20414-1651$ b & $1.37( \pm 0.47)$ & 0.67 & 3 & $\ldots$ & 16.2 & $15.7^{\mathrm{h}}$ \\
\hline $20551-4250$ & $1.32( \pm 0.25)$ & 0.113 & 84 & $3.3^{\mathrm{g}}$ & $\ldots$ & $16.9^{\mathrm{j}, h}$ \\
\hline $21219-1757 \mathrm{~b}$ & $4.19( \pm 3.29)$ & 0.14 & -50 & $\ldots$ & 16.9 & $15.6^{\mathrm{h}}$ \\
\hline $21504-0628$ & $1.95( \pm 0.26)$ & 0.164 & 12 & $\ldots$ & $\ldots$ & $\ldots$ \\
\hline $23230-6926$ & $2.03( \pm 0.25)$ & 0.177 & 41 & $\ldots$ & $16.9^{\mathrm{i}}$ & $16.2^{\mathrm{j}, h}$ \\
\hline $23365+3604$ & $4.8( \pm 1.0)^{\mathrm{e}}$ & $\ldots$ & $\ldots$ & $\ldots$ & $\ldots$ & $\ldots$ \\
\hline $23578-5307$ & $3.96( \pm 1.36)$ & 0.447 & -80 & $\ldots$ & $\ldots$ & $\ldots$ \\
\hline $\operatorname{Arp} 220^{\mathrm{d}}$ & 3.00 & $\ldots$ & $\ldots$ & $0.69( \pm 0.03)^{\mathrm{c}}$ & $17.4^{\mathrm{d}, h}$ & $16.9^{\mathrm{d}, h}$ \\
\hline NGC $6240^{\mathrm{d}}$ & 1.44 & $\ldots$ & $\ldots$ & $0.92( \pm 0.23)^{\mathrm{c}}$ & $14.3^{\mathrm{d}, h}$ & $13.8^{\mathrm{d}, h}$ \\
\hline $00456-2904(\mathrm{SW})$ & $2.09( \pm 0.15)$ & 0.067 & -88 & $\ldots$ & 16.3 & 14.9 \\
\hline 09111-1007(W) & $2.18( \pm 0.36)$ & 0.397 & -26 & $\ldots$ & $\ldots$ & $\ldots$ \\
\hline $14348-1447(\mathrm{NE})$ & $3.03( \pm 0.50)$ & 0.237 & 4 & $3( \pm 1)^{\mathrm{e}}$ & $18.3^{\mathrm{j}}$ & $17.5^{\mathrm{j}}$ \\
\hline 14348-1447(SW) & $2.78( \pm 0.03)$ & 0.113 & 7 & $3( \pm 1)^{\mathrm{e}}$ & $17.8^{\mathrm{j}}$ & $16.8^{j}$ \\
\hline
\end{tabular}

Note.-Structural and photometric properties of (mainly) ULIRG remnants. In this table we present data for newly observed sources during our VLT large program and we summarize observations carried out during our older normal programs (Genzel et al. 2001; Tacconi et al. 2002) that are used in this work. The sources in this Table together with those presented in Paper I summarize the 54 sources in our sample. The $H$-band structural parameters $R_{\text {eff }}$, ellipticity, and $\phi_{\alpha}$ of the sources are derived from the ISAAC acquisition images unless otherwise noted in the first column. The angle $\phi_{\alpha}$ is tabulated here only for the first slit; for the second slit it can be computed using the angle between the two slit positions given in Table 1. The galaxy surface brightness within the effective radius, $\mu_{\text {gal }}$ is derived from Veilleux et al. (2006) and Kim et al. (2002) respectively, unless otherwise noted. The $H$ - and $K$-band $\mu_{\text {gal }}$ data are presented after subtraction of the nuclear PSF for both bands; we indicate when individual PSF and galaxy magnitudes are not available in the literature. All data presented in this Table are derived from maximum-aperture photometry (enclosing the whole galaxy) and are not corrected for extinction effects.

${ }^{a}$ For the sources observed using NIRSPEC and the slit monitoring camera, the structural parameters are not extracted; the slit projection drawn on the acquisition image does not allow for photometric analysis. 
${ }^{b}$ Data taken from H-band NICMOS imaging by Veilleux et al. (2006). All quantities are PSF-subtracted and are converted to our cosmology.

${ }^{\mathrm{c}}$ Structural parameters extracted from NICMOS imaging data, kindly provided to us by Nick Scoville.

${ }^{\mathrm{d}}$ Based on Scoville et al. (2000).

eEffective radius from Genzel et al. (2001) or Tacconi et al. (2002).

${ }^{\mathrm{f}}$ Data from the 1 Jy sample analysis (Veilleux et al. 2002).

gEffective radius from Rothberg \& Joseph (2004).

${ }^{\mathrm{h}}$ For these sources the relative strength of the PSF and the Sersic component fit to the underlying galaxy are not known. We assume that $L_{\text {gal }}(H$ band $)=0.64 L_{\text {tot }}(H$ band $)$ and that $L_{\text {gal }}(K$ band $)=$ $0.48 L_{\text {tot }}(K$ band), (Colina et al. 2001; Surace \& Sanders 1999; Veilleux et al. 2006).

${ }^{\mathrm{i}} H$-band magnitude taken from Colina et al. (2001).

${ }^{\mathrm{j}}$ magnitude taken from Duc et al. (1997).

${ }^{\mathrm{k}} K$-band magnitude taken from Surace \& Sanders (1999).

${ }^{1} K$-band magnitude taken from Rigopoulou et al. (1999). 
TABLE 3

Stellar VELOCities AND BLACK HOLE MASSES

\begin{tabular}{|c|c|c|c|c|c|c|c|c|c|}
\hline $\begin{array}{l}\text { Source } \\
\text { (IRAS) }\end{array}$ & $\left(\begin{array}{c}\sigma \\
\left(\mathrm{km} \mathrm{s}^{-1}\right) \\
\end{array}\right.$ & $\begin{array}{c}\text { aper. }^{\mathrm{a}} \\
\left({ }^{\prime \prime}\right) \\
\end{array}$ & $\begin{array}{l}\text { aper. }^{a} \\
(\mathrm{kpc})\end{array}$ & $\begin{array}{l}V_{\text {rot }}(\text { obs }) \\
\left(\mathrm{km} \mathrm{s}^{-1}\right)\end{array}$ & $\begin{array}{c}\text { aper. }^{c} \\
\left({ }^{\prime \prime}\right) \\
\end{array}$ & $V_{\operatorname{rot}}(\mathrm{obs}) / \sigma$ & $\begin{array}{l}M_{\mathrm{BH}} \mathrm{d} \\
\left(M_{\odot}\right) \\
\end{array}$ & $\begin{array}{c}M_{\mathrm{BH}}(\text { Edd. }) \\
\left(M_{\odot}\right) \\
\end{array}$ & $\eta_{\text {Edd }}$ \\
\hline $00091-0738$ & $131( \pm 39)$ & 0.45 & 1.1 & $\cdots$ & $\ldots$ & $\ldots$ & $2.46 \times 10^{7}$ & $2.04 \times 10^{7}$ & 0.83 \\
\hline $00262+4251$ & $170( \pm 15)$ & $\ldots$ & $\ldots$ & $<15$ & $\cdots$ & $<0.09$ & $7.02 \times 10^{7}$ & $1.32 \times 10^{7}$ & 0.19 \\
\hline F01004-2237 & $132( \pm 29)$ & 0.29 & 0.7 & $22( \pm 13)$ & 0.60 & 0.17 & $2.54 \times 10^{7}$ & $2.29 \times 10^{7}$ & 0.90 \\
\hline $01388-4618$ & $144( \pm 10)$ & $\ldots$ & . . & $130( \pm 15)$ & $\ldots$ & 0.90 & $3.60 \times 10^{7}$ & $1.32 \times 10^{7}$ & 0.37 \\
\hline $01572+0009$ & $200( \pm 60)$ & $\ldots$ & $\ldots$ & $\ldots$ & $\ldots$ & $\ldots$ & $1.35 \times 10^{8}$ & $4.46 \times 10^{7}$ & 0.33 \\
\hline F02021-2103 & $143( \pm 21)$ & 0.37 & 0.9 & $42( \pm 10)$ & 0.76 & 0.29 & $3.50 \times 10^{7}$ & $1.35 \times 10^{7}$ & 0.38 \\
\hline $05189-2524$ & $137( \pm 16)$ & 0.29 & 0.3 & $70( \pm 14)$ & 0.51 & 0.51 & $2.95 \times 10^{7}$ & $1.62 \times 10^{7}$ & 0.55 \\
\hline $09039+0503$ & $183( \pm 38)$ & 0.58 & 1.5 & $\ldots$ & $\ldots$ & $\ldots$ & $9.45 \times 10^{7}$ & $1.55 \times 10^{7}$ & 0.16 \\
\hline $11223-1244$ & $149( \pm 27)$ & 0.73 & 3.0 & $\ldots$ & $\ldots$ & $\ldots$ & $4.13 \times 10^{7}$ & $5.12 \times 10^{7}$ & 1.24 \\
\hline $12540+5708$ & $120( \pm 10)$ & $\cdots$ & $\ldots$ & $25( \pm 10)$ & $\ldots$ & 0.21 & $1.73 \times 10^{7}$ & $4.16 \times 10^{7}$ & 2.40 \\
\hline $13428+5608$ & $285( \pm 30)$ & $\ldots$ & $\ldots$ & $110( \pm 20)$ & $\ldots$ & 0.39 & $5.61 \times 10^{8}$ & $1.66 \times 10^{7}$ & 0.03 \\
\hline $14070+0525$ & $139( \pm 21)$ & 0.70 & 3.8 & $54( \pm 19)$ & 0.77 & 0.39 & $3.13 \times 10^{7}$ & $7.57 \times 10^{7}$ & 2.42 \\
\hline $14378-3651$ & $153( \pm 10)$ & $\ldots$ & $\ldots$ & $15( \pm 10)$ & $\ldots$ & 0.10 & $4.60 \times 10^{7}$ & $1.66 \times 10^{7}$ & 0.36 \\
\hline $20414-1651$ & $187( \pm 32)$ & 0.44 & 0.8 & $96( \pm 38)$ & 0.88 & 0.51 & $1.03 \times 10^{8}$ & $1.82 \times 10^{7}$ & 0.18 \\
\hline $20551-4250$ & $140( \pm 15)$ & $\cdots$ & $\ldots$ & $40( \pm 10)$ & $\cdots$ & 0.29 & $3.22 \times 10^{7}$ & $1.32 \times 10^{7}$ & 0.41 \\
\hline $21219-1757$ & $121( \pm 11)$ & 0.58 & 1.3 & $\ldots$ & $\ldots$ & $\ldots$ & $1.79 \times 10^{7}$ & $1.51 \times 10^{7}$ & 0.84 \\
\hline $21504-0628$ & $90( \pm 31)$ & 0.32 & 0.5 & $9( \pm 28)$ & 0.48 & 0.10 & $5.45 \times 10^{6}$ & $8.30 \times 10^{6}$ & 1.52 \\
\hline $23230-6926$ & $143( \pm 14)$ & 0.48 & 1.1 & $23( \pm 13)$ & 0.73 & 0.16 & $3.50 \times 10^{7}$ & $1.44 \times 10^{7}$ & 0.41 \\
\hline $23365+3604$ & $145( \pm 15)$ & $\ldots$ & $\ldots$ & $<15$ & $\ldots$ & $<0.10$ & $3.71 \times 10^{7}$ & $1.62 \times 10^{7}$ & 0.44 \\
\hline $23578-5307$ & $190( \pm 70)$ & $\ldots$ & $\ldots$ & $\ldots$ & $\ldots$ & $\ldots$ & $1.10 \times 10^{8}$ & $1.66 \times 10^{7}$ & 0.15 \\
\hline Arp 220 & $164( \pm 10)$ & $\ldots$ & $\ldots$ & $185( \pm 30)^{g}$ & $\ldots$ & 0.84 & $6.08 \times 10^{7}$ & $1.66 \times 10^{7}$ & 0.27 \\
\hline NGC 6240 & $229( \pm 43)^{h}$ & $\cdots$ & $\ldots$ & $240( \pm 108)^{h}$ & $\ldots$ & 1.05 & $2.33 \times 10^{8}$ & $8.30 \times 10^{6}$ & 0.04 \\
\hline $00456-2904(\mathrm{SW})$ & $162( \pm 25)$ & $\ldots$ & $\ldots$ & $45( \pm 10)$ & $\ldots$ & 0.28 & $5.79 \times 10^{7}$ & $1.73 \times 10^{7}$ & 0.30 \\
\hline $09111-1007(\mathrm{~W})$ & $112( \pm 18)$ & 0.29 & 0.3 & $68( \pm 16)$ & 1.17 & 0.61 & $1.31 \times 10^{7}$ & $9.53 \times 10^{6}$ & 0.73 \\
\hline $14348-1447(\mathrm{NE})$ & $170( \pm 14)$ & $\cdots$ & $\ldots$ & $60( \pm 20)$ & $\ldots$ & 0.35 & $7.02 \times 10^{7}$ & $1.92 \times 10^{7}$ & 0.27 \\
\hline $14348-1447(\mathrm{SW})$ & $150( \pm 25)$ & $\ldots$ & $\ldots$ & $50( \pm 15)$ & $\ldots$ & 0.33 & $4.25 \times 10^{7}$ & $3.33 \times 10^{7}$ & 0.78 \\
\hline
\end{tabular}

NoTE. - The stellar central velocity dispersion, rotational velocity, and the $V_{\text {rot }} / \sigma$ ratio of the newly-observed sources are derived from the the two are also presented here.

${ }^{a}$ Radial extent of the central aperture used for the extraction of $\sigma$, tabulated both in angular and linear distances.

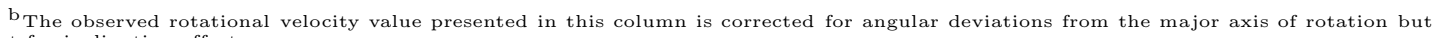
not for inclination effects.

${ }^{c}$ The center of the outer aperture used for the extraction of $V_{\text {rot }}$. The annular width of this aperture is equal to the radius of the corresponding central aperture given in the third column.

d Dynamical black hole masses estimated from their relation to the bulge dispersion (Tremaine et al. 2002).

${ }^{e}$ Eddington black hole mass, calculated by attributing $50 \%$ of $\mathrm{L}_{\mathrm{IR}}$ to the AGN.

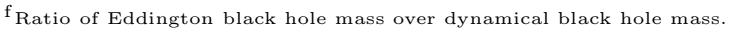

${ }^{g}$ We use as rotational velocity of Arp 220 that of the east component, since that of the west component is only a lower limit (Genzel et al. 2001).

${ }^{\mathrm{h}}$ Data are taken from Tecza et al. (2000). The average of the velocity dispersion values at the two nuclei and the internuclear region is tabulated here. This value is also close to the luminosity-weighted average of the two nuclei which equals $225 \mathrm{~km} \mathrm{~s}^{-1}$. 
IRAS $00091-0738$

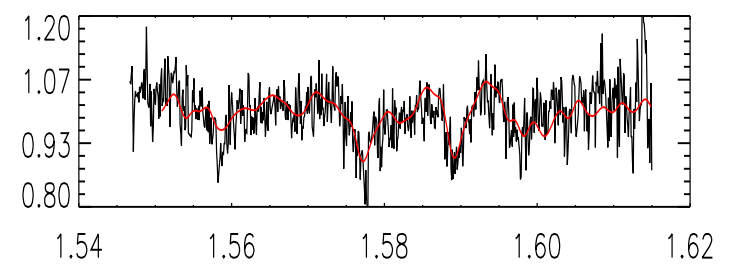

IRAS 01004-2237
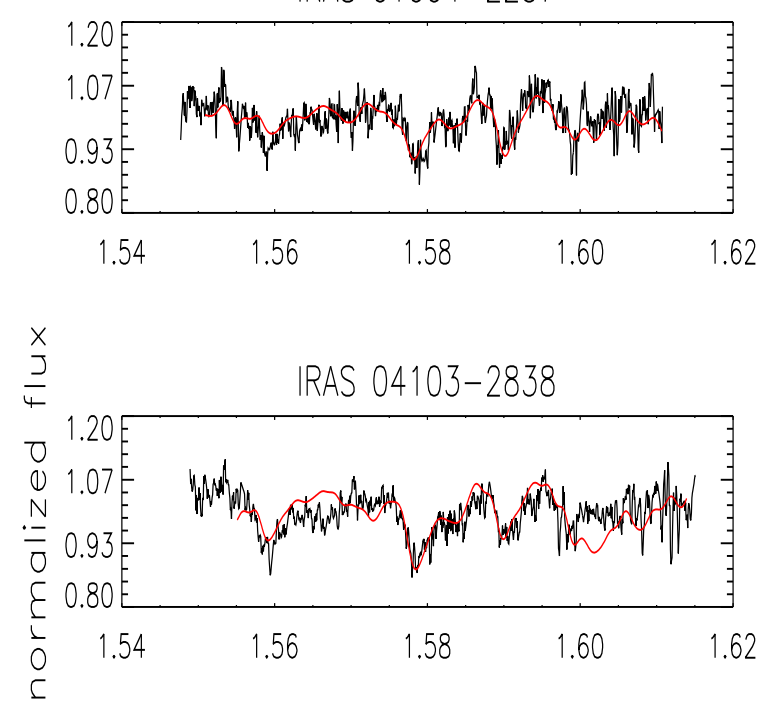

IRAS 05189-2524

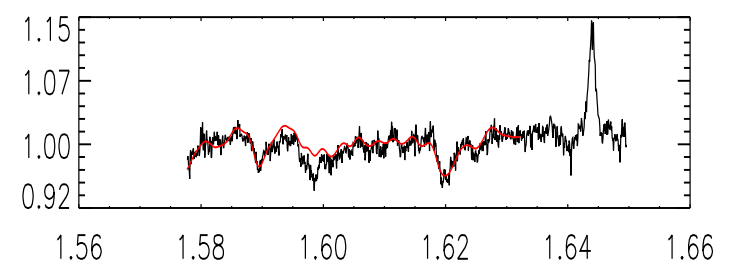

IRAS $09111-1007$

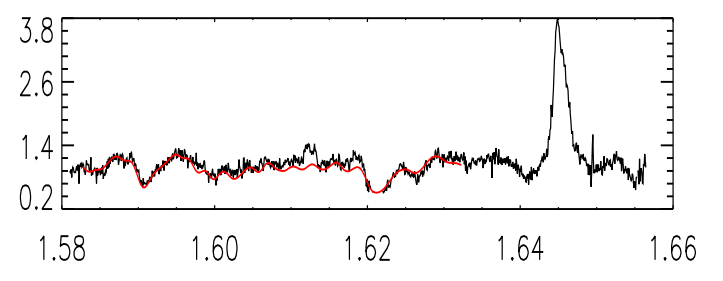

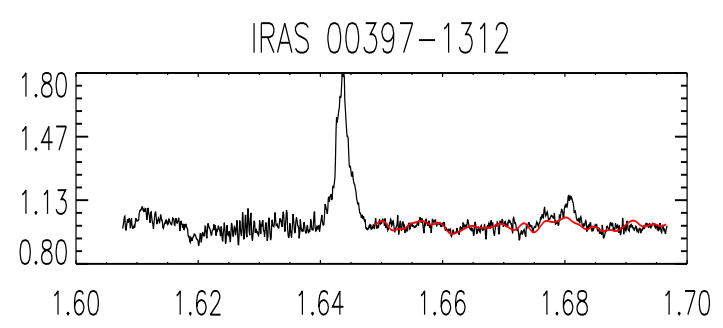
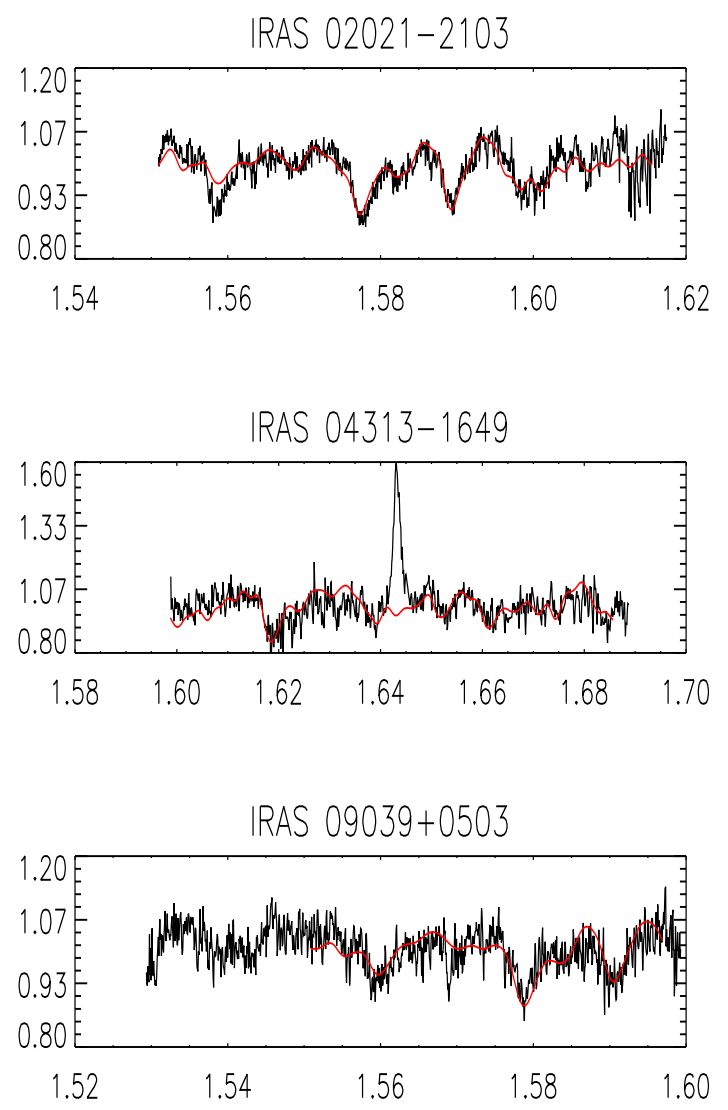

IRAS $11223-1244$

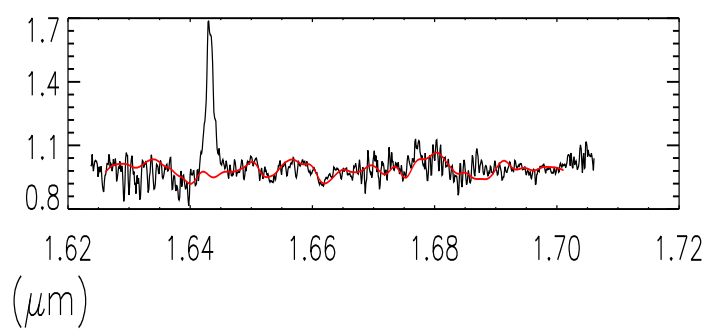

Fig. 1.- The $H$-band spectra of the ULIRG remnants from this study. The stellar template, convolved with Gaussians that represent the LOS broadening function of the sources, is overplotted as a solid line. All spectra are shifted to rest frame. 
IRAS $14070+0525$

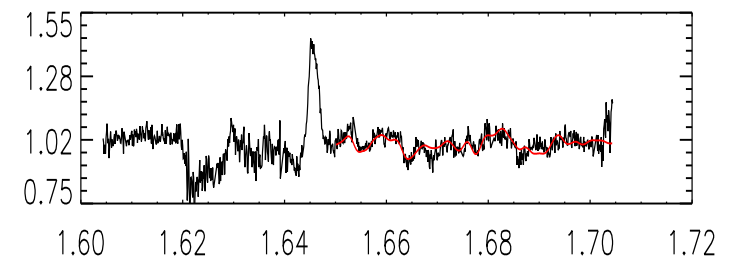

IRAS 15462-0459

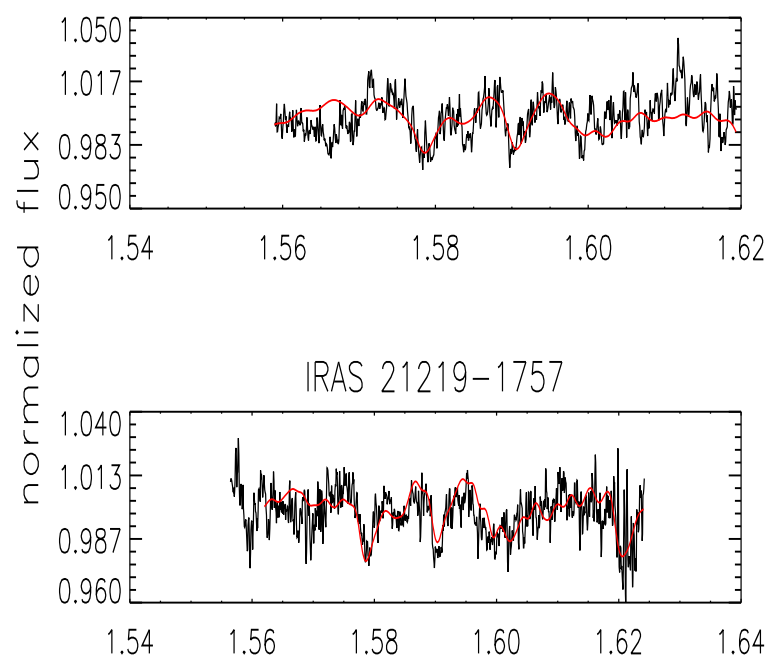

IRAS 23230-6926

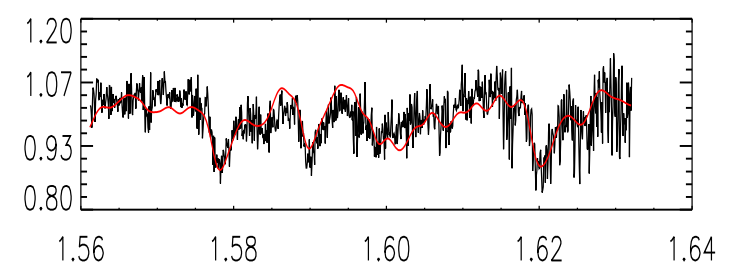

IRAS 15130-1958

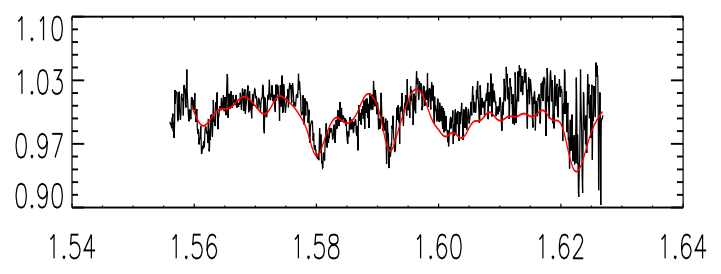

IRAS 20414-1651

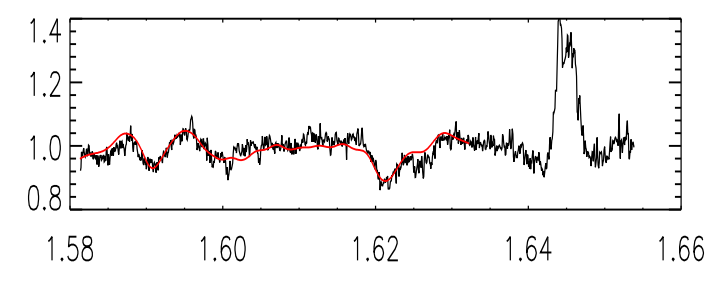

IRAS 21504-0628

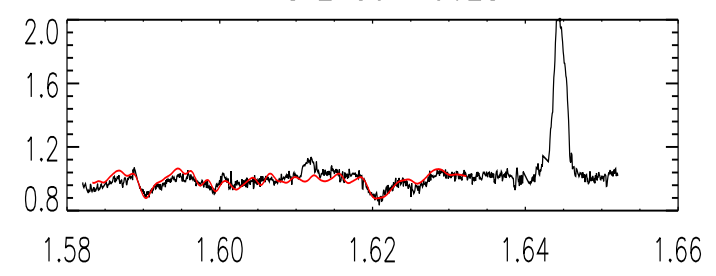

wavelength ( $\mu m)$

Fig. 1 continued. 

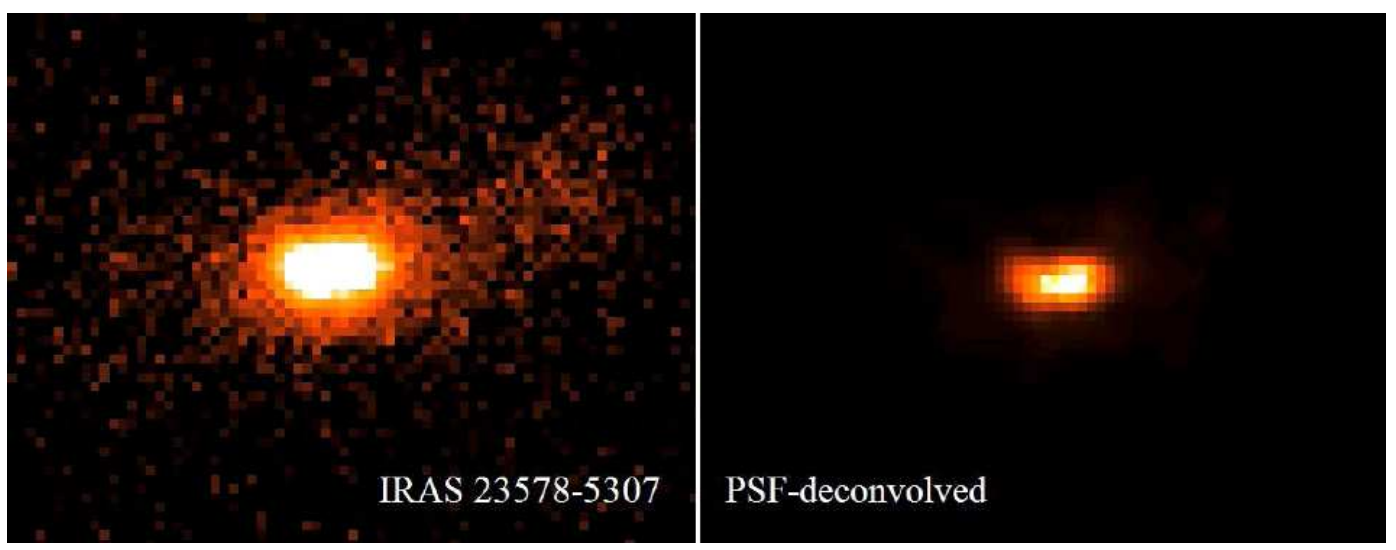

Fig. 2.- The ISAAC $H$-band raw (left panel) and PSF-deconvolved (right panel) acquisition image of IRAS $23578-5307$.
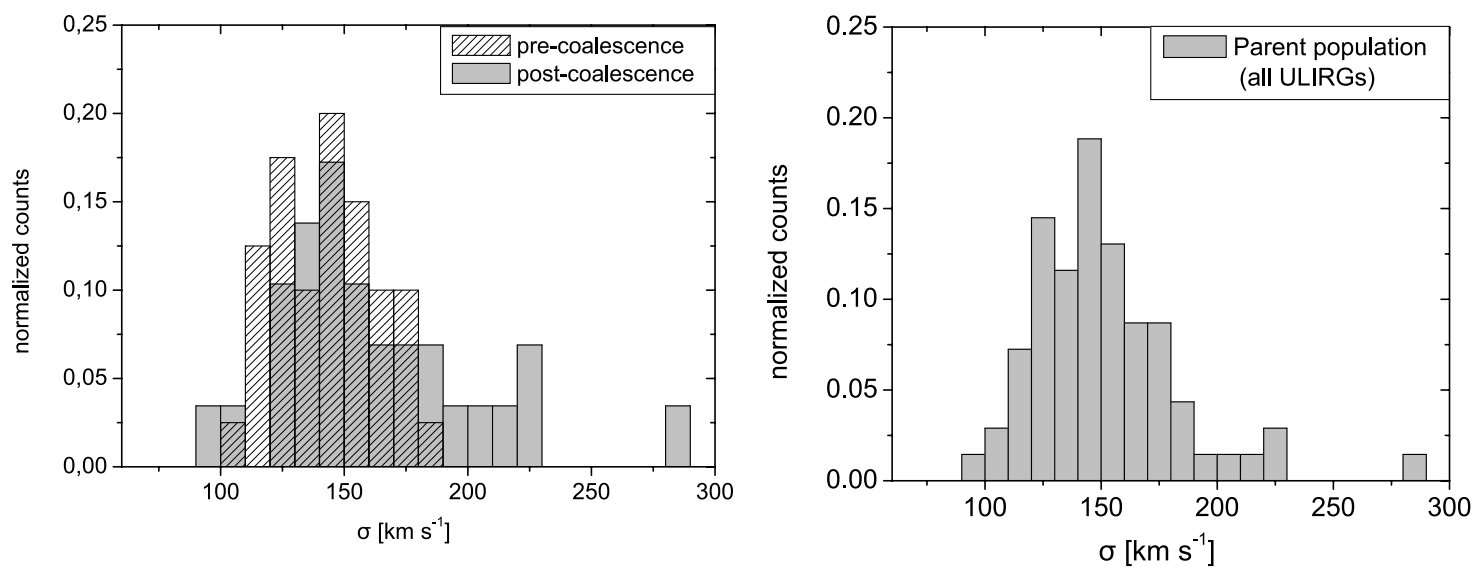

Fig. 3.- Left panel: The distributions of stellar dispersions in ULIRGs before and after nuclear coalescence. Right panel: The ULIRG parent-population distribution used in our Monte Carlo simulations. 

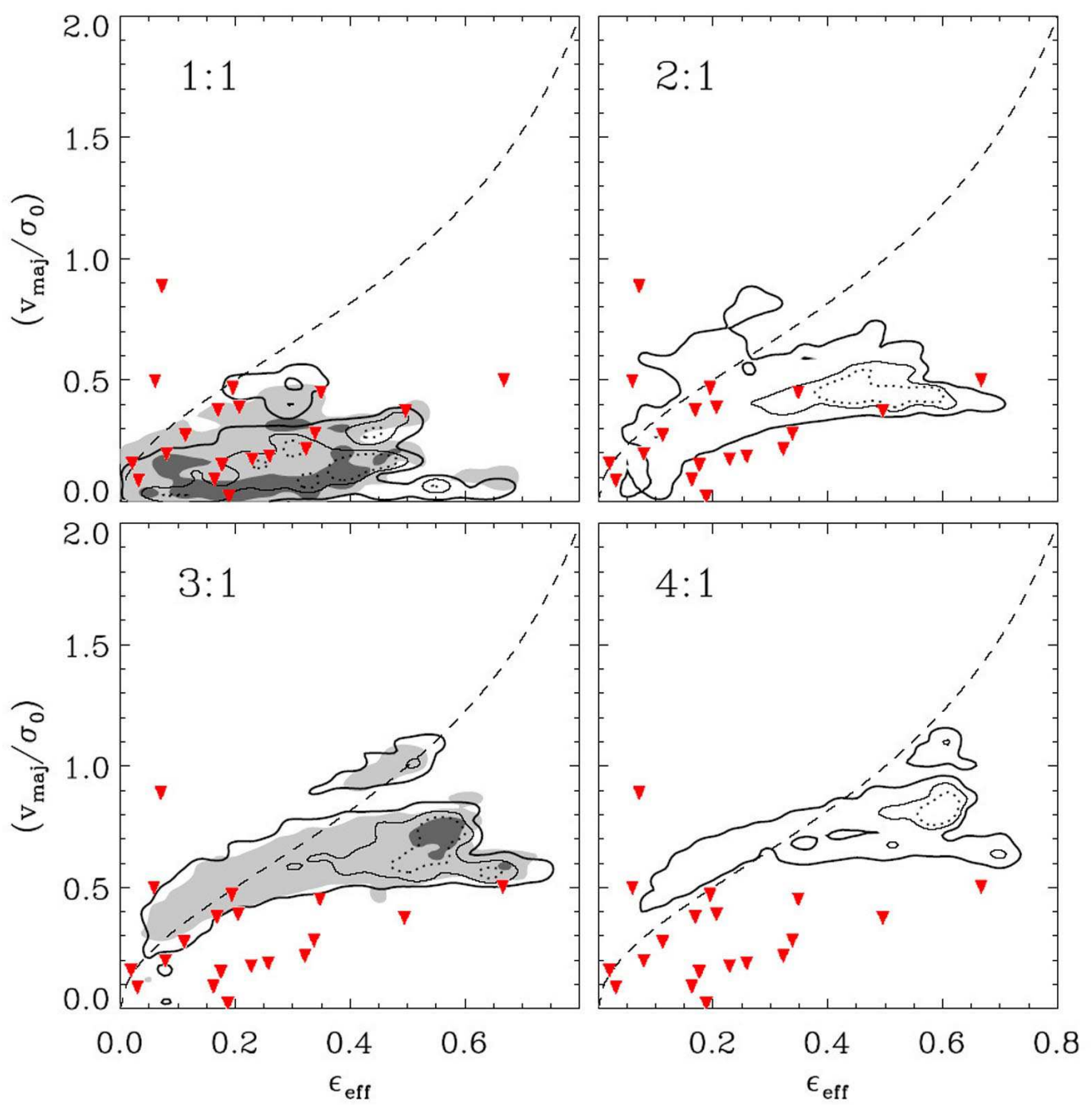

Fig. 4.- Ratio of observed rotational over dispersion velocity versus ellipticity for merger remnants (from Naab \& Burkert 2003 and Naab et al. 2006b). Each panel corresponds to mergers of different progenitor mass ratios (cases from 1:1 to 4:1 are studied). The bold line, solid line, and dotted contours correspond respectively to the $90 \%, 70 \%$, and $50 \%$ probability of finding a collisionless merger remnant in the enclosed region. The dark- and light-gray shaded areas indicate the region in the diagram where gas-rich merger remnants are expected to be found at $90 \%$ and $50 \%$ probability levels. The dashed line shows the theoretical values for an oblate isotropic rotator. ULIRG remnants are shown as triangles. 


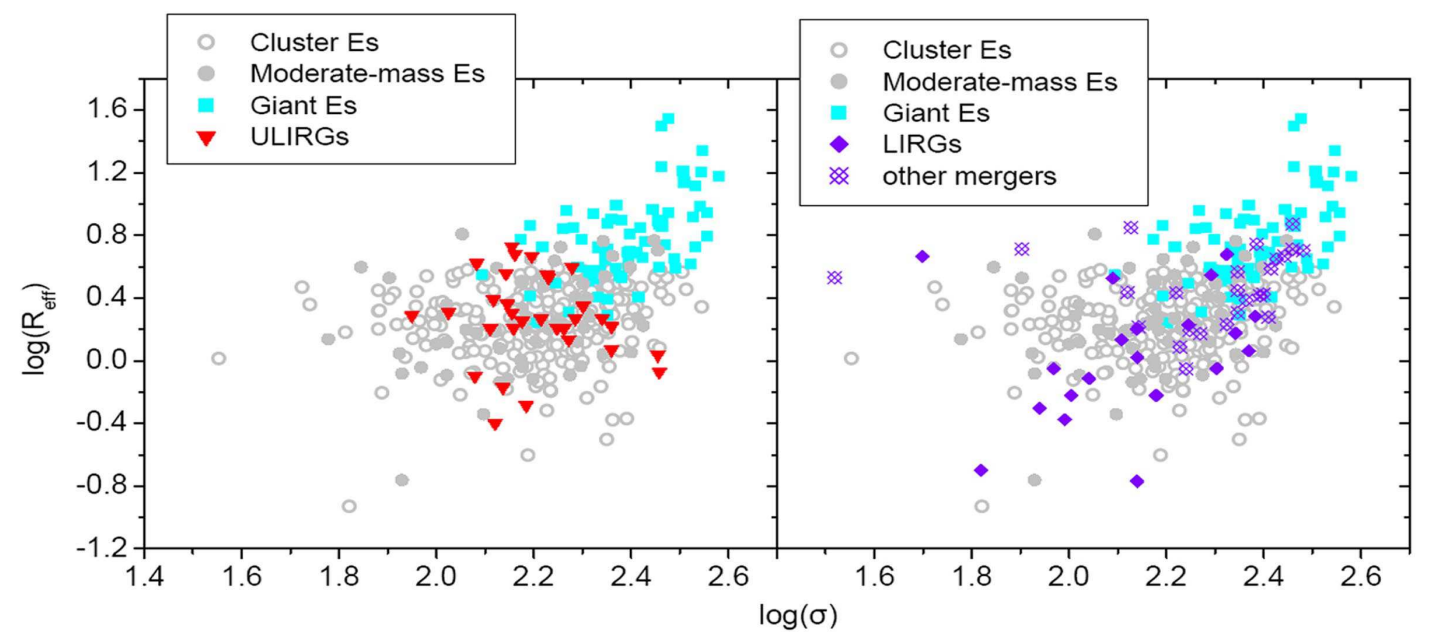

Fig. 5.- The $R_{\mathrm{eff}}-\sigma$ projection of the early-type galaxy fundamental plane. The data for the giant boxy and moderate-mass disky Es (squares and circles respectively) are taken from Bender et al. (1992) and Faber et al. (1997). More (cluster) Es (open circles) are from Pahre (1999). For viewing clarity, the various types of mergers are plotted in separate panels. The ULIRG remnants (29 from this study and 2 from Rothberg \& Joseph 2006a) are plotted as triangles (left panel). LIRGs (diamonds) from Shier \& Fischer (1998), James et al. (1999), Rothberg \& Joseph (2006a), and Hinz \& Rieke (2006) and other (visually-selected) mergers from Rothberg \& Joseph (2006a) are plotted in diamonds and open-crossed diamonds respectively (right panel). The effective radii of all merger remnants used in this figure are equal to the averages of their NIR measurements, if more than one is available. 

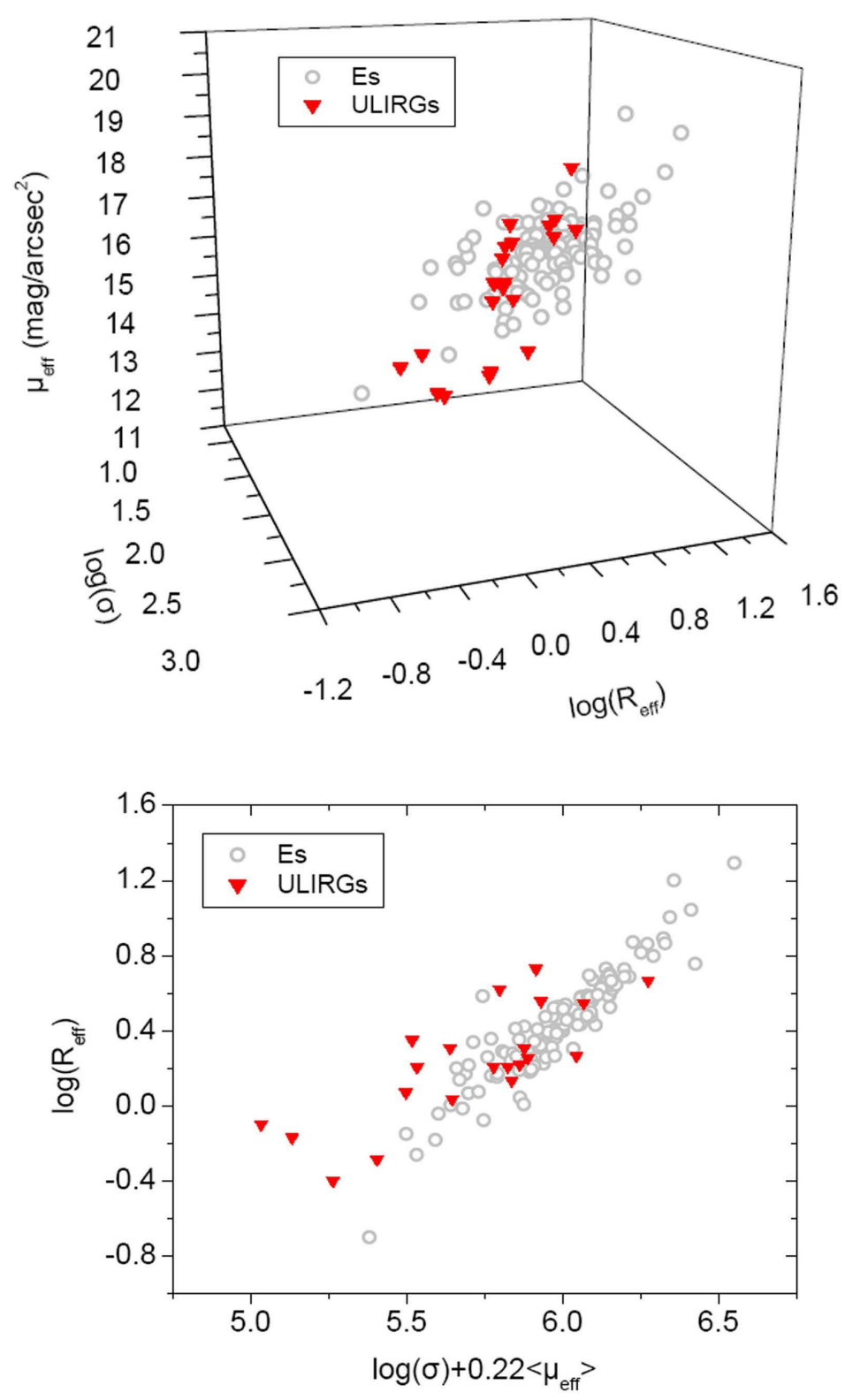

Fig. 6.- Upper panel: The 3-dimensional view of the $H$-band fundamental plane of early-type galaxies. Local early-type galaxies (mainly from the Coma and Virgo clusters) are plotted as open circles (Zibetti et al. 2002). 21 ULIRGs from this study are plotted as triangles. All data are PSF removed (see Table 2). No extinction corrections have been applied to the fluxes. The effective radii used are as in Fig. 5 Lower panel: The fundamental plane, viewed as in Pahre (1999). 

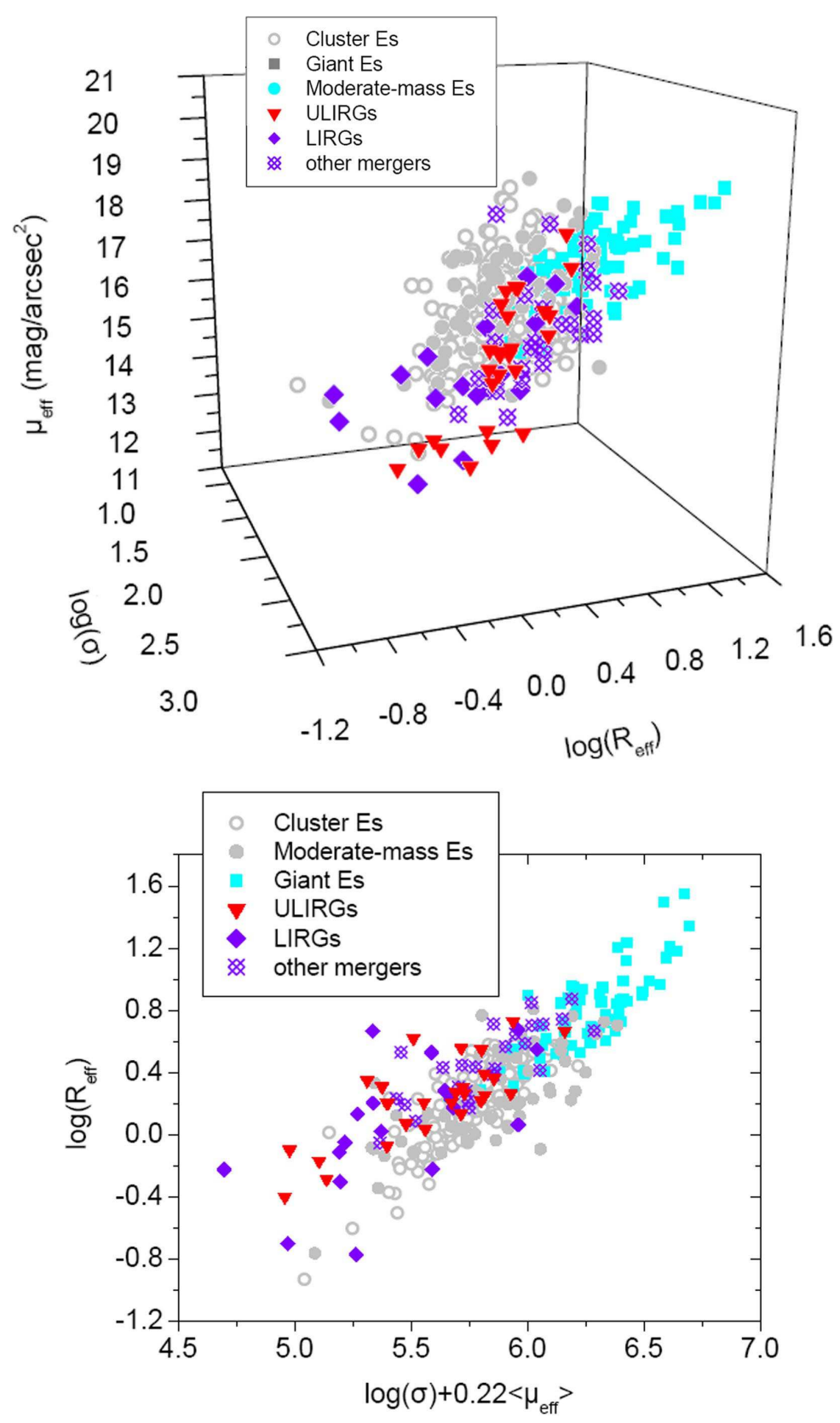

Fig. 7.- Upper panel: The 3-dimensional view of the $K$-band fundamental plane of early-type galaxies. The symbols and effective radii used are identical to those in Fig. 5. A PSF estimate has been removed to all photometric data initially taken from Kim et al. (2002) (see Table 2). No extinction corrections have been applied to the data. From the ULIRGs plotted in this figure, 25 are from this study and 2 from Rothberg \& Joseph (2006a). Lower panel: The fundamental plane, viewed as in Pahre (1999). 


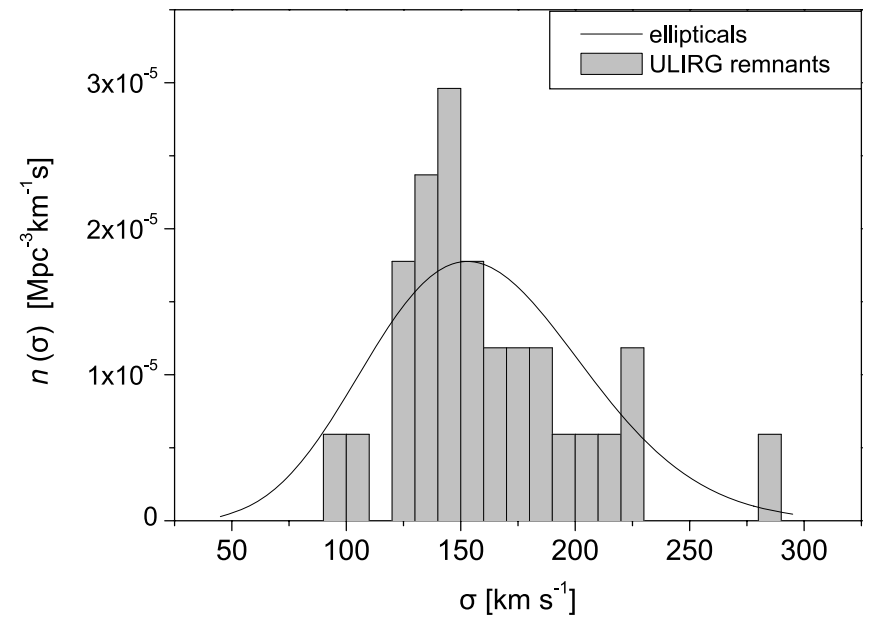

Fig. 8.- The number density of sources as a function of their stellar velocity dispersion is plotted in this figure. The solid line corresponds to the number density per $\sigma$ of SDSS ellipticals, computed from their dispersion function (Sheth et al. 2003). The number density per $\sigma$ of ULIRG remnants, plotted as a histogram, is calculated by multiplying the \% fraction of our remnants that resides in each $\sigma$ bin with the local volume density of ULIRGs from Sanders et al. (2003). To facilitate the comparison, the ULIRG histogram is further normalized so that its mean has the same number density as that of the elliptical $n(\sigma)$ distribution. 

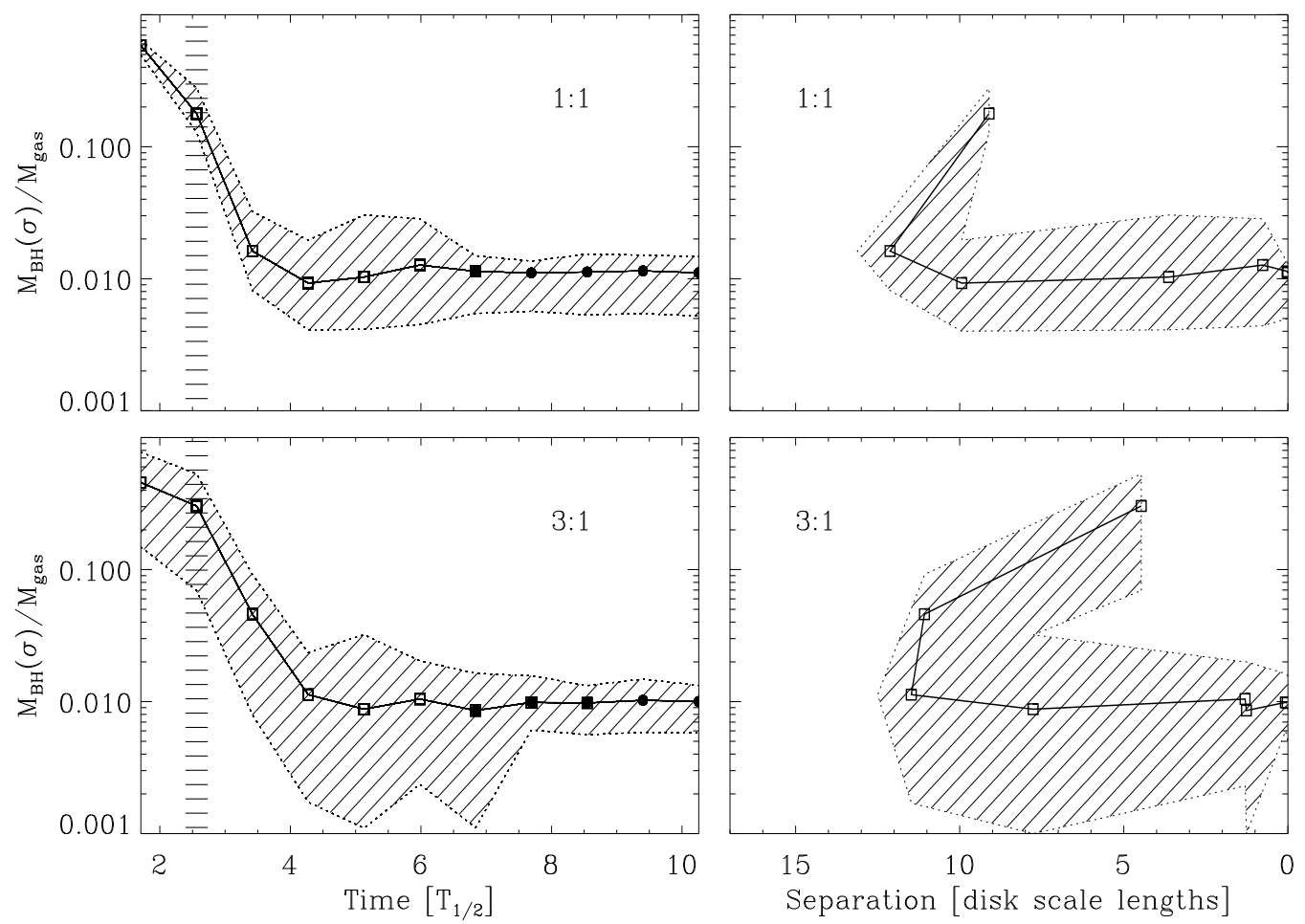

Fig. 9.- Evolution, during a disk-galaxy merger, of the average black-hole mass inferred from the central (line-of-sight) stellar velocity dispersion of the simulated galaxies. The black hole mass is in units of the total gas mass accreted onto the center of the simulations. In the left column, the evolution is given as a function of time. Time is plotted in units of the half-mass rotation period, $T_{1 / 2}$, of the more massive progenitor disk. Open squares represent disks that are still separated, filled dots indicate fully merged systems. The spread originating from the initial disk orientations is indicated by the diagonally shaded area. The vertically shaded area indicates the first data point after the first encounter. In the right column, the evolution is plotted as a function of nuclear separation of the interacting galaxies. The nuclear separation unit is the disk scale length $h$ of the more massive disk. We begin plotting data points for 1 step prior to first encounter, as in the left panels. We show 1:1 mergers in the upper and 3:1 mergers in the lower panels. 